\title{
Examples of Riemannian Manifolds with Non-negative Sectional Curvature
}

\author{
Wolfgang Ziller
}

Manifolds with non-negative sectional curvature have been of interest since the beginning of global Riemannian geometry, as illustrated by the theorems of Bonnet-Myers, Synge, and the sphere theorem. Some of the oldest conjectures in global Riemannian geometry, as for example the Hopf conjecture on $\mathbb{S}^{2} \times \mathbb{S}^{2}$, also fit into this subject.

For non-negatively curved manifolds, there are a number of obstruction theorems known, see Section 1 below and the survey by Burkhard Wilking in this volume. It is somewhat surprising that the only further obstructions to positive curvature are given by the classical Bonnet-Myers and Synge theorems on the fundamental group.

Although there are many examples with non-negative curvature, they all come from two basic constructions, apart from taking products. One is taking a quotient of a compact Lie group with a biinvariant metric and another a gluing procedure due to Cheeger and recently significantly generalized by Grove-Ziller. The latter examples include a rich class of manifolds, and give rise to non-negative curvature on many exotic 7 -spheres. On the other hand, known manifolds with positive sectional curvature are very rare, and are all given by quotients of compact Lie groups, and, apart from the classical rank one symmetric spaces, only exist in dimension below 25 .

Due to this lack of knowledge, it is therefore of importance to discuss and understand known examples and find new ones. In this survey we will concentrate on the description of known examples, although the last section also contains suggestions where to look for new ones. The techniques used to construct them are fairly simple. In addition to the above, the main tool is a deformation described by Cheeger that, when applied to non-negatively curved manifolds, tends to increase curvature. Such Cheeger deformations can be considered as the unifying theme of this survey. We can thus be fairly explicit in the proof of the existence of all known examples which should make the basic material understandable at an advanced graduate student

The author was supported by a grant from the National Science Foundation. 
level. It is the hope of this author that it will thus encourage others to study this beautiful subject. This survey originated in the Rudolph Lipschitz lecture series the author gave at the University of Bonn in 2001 and various courses taught at the University of Pennsylvania.

\section{General structure theorems}

To put the examples discussed in this survey into context, we mention the main structure theorems and conjectures in this subject. See the survey by Burkhard Wilking in this volume for further information.

- (Gromov) If $M^{n}$ is a compact manifold with sec $\geq 0$, then there exists a universal constant $c(n)$ such that $b_{i}\left(M^{n}, F\right) \leq c(n)$ for all $i$ and any field of coefficients $F$. Furthermore, the fundamental group has a generating set with at most $c(n)$ elements.

- (Cheeger-Gromoll) If $M^{n}$ is a compact manifold that admits a metric with non-negative sectional curvature, then there exists an abelian subgroup of $\pi_{1}\left(M^{n}\right)$ with finite index.

- (Lichnerowicz-Hitchin) The obstructions to positive scalar curvature imply that a compact spin manifold with $\hat{A}(M) \neq 0$ or $\alpha(M) \neq$ 0 does not admit a metric with non-negative sectional curvature. This holds in particular for the unique exotic sphere in dimension 9.

- (Cheeger-Gromoll) If $M^{n}$ is a non-compact manifold with a complete metric with sec $\geq 0$, then there exists a totally geodesic compact submanifold $S^{k}$, called the soul, such that $M^{n}$ is diffeomorphic to the normal bundle of $S^{k}$.

Surprisingly, for positive curvature one has in addition only the classical obstructions:

- (Bonnet-Myers) A manifold which admits a metric with positive curvature has finite fundamental group.

- (Synge) An even dimensional manifold with positive curvature has fundamental group 0 , if orientable, and $\mathbb{Z}_{2}$, if non-orientable. In odd dimensions a positively curved manifold is orientable.

If we allow ourselves to add an upper as well as a lower bound on the sectional curvature it is convenient to introduce what is called the pinching constant which is defined as $\delta=\min \mathrm{sec} / \mathrm{max} s e c$. One then has the following recognition and finiteness theorems:

- (Berger-Klingenberg) If $M^{n}$ is a compact simply connected manifold with $\delta \geq \frac{1}{4}$, then $M$ is either homeomorphic to $\mathbb{S}^{n}$ or isometric to $\mathbb{C} \mathbb{P}^{n}, \mathbb{H} \mathbb{P}^{n}$ or $\mathrm{CaP}^{2}$ with their standard Fubini metric.

- (Cheeger) Given a positive constant $\epsilon$, there are only finitely many diffeomorphism types of compact simply connected manifolds $M^{2 n}$ with $\delta \geq \epsilon$. 
- (Fang-Rong, Petrunin-Tuschmann) Given a positive constant $\epsilon$, there are only finitely many diffeomorphism types of compact manifolds $M^{2 n+1}$ with $\pi_{1}(M)=\pi_{2}(M)=0$ and $\delta \geq \epsilon$.

We finally mention some conjectures.

- (Hopf) There exists no metric with positive sectional curvature on $\mathbb{S}^{2} \times \mathbb{S}^{2}$. More generally, there are no positively curved metrics on the product of two compact manifolds, or on a symmetric space of rank at least two.

- (Hopf) A compact manifold with sec $\geq 0$ has non-negative Euler characteristic. An even dimensional manifold with positive curvature has positive Euler characteristic.

- (Bott) A compact simply connected manifold $M$ with sec $\geq 0$ is elliptic, i.e., the sequence of Betti numbers of the loop space of $M$ grows at most polynomially for every field of coefficients.

The latter conjecture, and its many consequences, were discussed in the literature for the first time in $[\mathbf{3 7}]$.

\section{Compact examples with non-negative curvature}

There are two natural constructions to produce new metrics with nonnegative curvature from given ones. If $M_{1}$ and $M_{2}$ are endowed with metrics of non-negative curvature, the product metric on $M_{1} \times M_{2}$ clearly has nonnegative curvature. The second construction is by taking quotients, or more generally by considering Riemannian submersions.

Recall that if $M$ and $B$ are two Riemannian manifolds, then a smooth map $\pi: M \rightarrow B$ is called a Riemannian submersion if $\pi_{*}$ is an isometry on horizontal vectors, i.e., on vectors orthogonal to the fibers. For such submersions one has the O'Neill formula:

$$
\sec _{B}\left(\pi_{*} x, \pi_{*} y\right)=\sec _{M}(x, y)+\frac{3}{4}\left\|[X, Y]^{v}\right\|^{2},
$$

where $x, y$ are orthonormal horizontal vectors, i.e., orthogonal to the fibers, $X, Y$ are horizontal vector fields extending $x, y$, and $[X, Y]^{v}$ denotes the vertical part of $[X, Y]$, i.e., the component tangent to the fiber. If $M$ has non-negative curvature, so does $B$, and one can hope that in some cases $B$ is even positively curved. The most basic examples of Riemannian submersions are given by taking quotients $\pi: M \rightarrow M / G$ where $G$ is a compact Lie group acting freely and isometrically on $M$. We often call the induced metric on $M / G$ the 'quotient' metric.

Before we describe a third method, let us first recall some standard ways of putting metrics on homogeneous spaces. If a compact Lie group $G$ acts transitively on $M$ and $p \in M$, we can write $M=G / H$ where $H$ is the isotropy group at $p$. We will often fix a biinvariant metric $Q$ on $\mathfrak{g}$, the Lie algebra of $G$. Note that ad $X: Y \rightarrow[X, Y]$ is then a skew symmetric endomorphism with respect to $Q$. Thus the $Q$-orthogonal decomposition $\mathfrak{g}=\mathfrak{h}+\mathfrak{m}$ 
satisfies $[\mathfrak{h}, \mathfrak{h}] \subset \mathfrak{h}$ and $[\mathfrak{h}, \mathfrak{m}] \subset \mathfrak{m}$. The more restrictive condition $[\mathfrak{m}, \mathfrak{m}] \subset \mathfrak{h}$ corresponds to the case where the metric $Q$ induces a locally symmetric metric on $G / H$. We identify $\mathfrak{m}$ with $T_{p} M$ via action fields: $X \in \mathfrak{m} \rightarrow X^{*}(p)$ where $X^{*}(q)=\frac{d}{d t \mid t=0} \exp (t X) q$. The action of $H$ on $T_{p} M$ is then identified with the action of $\operatorname{Ad} H$ on $\mathfrak{m}$. A metric on $G / H$, invariant under the action of $G$, corresponds to an inner product on $\mathfrak{m} \cong T_{p} M$ which is $\operatorname{Ad}(H)$ invariant. This inner product can be expressed as $Q(P X, Y)$ where $X, Y \in \mathfrak{m}$ and $P: \mathfrak{m} \rightarrow \mathfrak{m}$ is a $Q$-symmetric endomorphism.

A third method that produces new non-negatively curved metrics from a given one is obtained via a Cheeger deformation. This process was first used by M. Berger who considered metrics on spheres, shrunk in the direction of the Hopf fibration, to produce odd dimensional manifolds with small injectivity radius and positively pinched curvature. A systematic general description was given in [18]. Let $(M, g)$ be a Riemannian manifold and $G$ a Lie group acting by isometries on $M$. We then consider the Riemannian submersion

$$
\pi: M \times G \rightarrow M \quad: \quad(p, g) \rightarrow g^{-1} p .
$$

This can also be viewed as a quotient construction via the action

$$
\tilde{g} \star(p, g)=(\tilde{g} p, \tilde{g} g) \text { or simply } M=(M \times G) / \triangle G .
$$

We can thus start with a non-negatively curved metric $g$ on $M$, take a product with a biinvariant metric, and then the quotient metric defines a new metric on $M$. To describe this process as a deformation, fix a biinvariant metric $Q$ on $\mathfrak{g}$ and let $g_{t}$ be the metric obtained as a quotient of the product metric $g+\frac{1}{t} Q$ on $M \times G$. Tangent to the orbit $G p=G / G_{p}$, we write the original metric as above in the form $Q(P X, Y)$, where $X, Y \in \mathfrak{m}_{p}$, with $\mathfrak{m}_{p}$ the orthogonal complement of the Lie algebra of $G_{p}$. The symmetric endomorphism $P: \mathfrak{m}_{p} \rightarrow \mathfrak{m}_{p}$ is changed into a new symmetric endomorphism $P_{t}$ describing $g_{t}$ in terms of $Q$ and we claim:

$$
P_{t}=\left(P^{-1}+t \mathrm{Id}\right)^{-1} \text {. }
$$

To see this, observe that $\pi_{*}\left(X^{*}, Y\right)=X^{*}-Y^{*}$. Thus a horizontal lift of $X \in \mathfrak{m}_{p} \cong T_{p}\left(G / G_{p}\right) \subset T_{p} M$, under the Riemannian submersion $\pi$, is equal to

$$
\left(P^{-1}\left(P^{-1}+t \mathrm{Id}\right)^{-1} X^{*}(p),-t\left(P^{-1}+t \mathrm{Id}\right)^{-1} X\right) \in \mathrm{T}_{p} M \times \mathfrak{g},
$$

and the length squared of this vector is

$$
\begin{aligned}
& Q \\
& \left.\quad\left(P^{-1}+t \mathrm{Id}\right)^{-1} X, P^{-1}\left(P^{-1}+t \mathrm{Id}\right)^{-1} X\right) \\
& \quad+\frac{1}{t} Q\left(t\left(P^{-1}+t \mathrm{Id}\right)^{-1} X, t\left(P^{-1}+t \mathrm{Id}\right)^{-1} X\right) \\
& \quad=Q\left(\left(P^{-1}+t \mathrm{Id}\right)^{-1} X, X\right) .
\end{aligned}
$$

Orthogonal to the orbit $G p$, the metric is unchanged since a horizontal lift of $X \in \mathfrak{m}_{p}^{\perp} \subset T_{p} M$ is equal to $\left(X^{*}(p), 0\right)$. 
This process can be considered as a deformation, since we obtain the original metric $g$ when $t=0$. If $\lambda_{i}$ are the eigenvalues of $P$, the eigenvalues of $P_{t}$ are $\frac{\lambda_{i}}{1+t \lambda_{i}}$, i.e. the metric is shrunk in the direction of the orbits. This process will in general destroy symmetries, although the group $G$ clearly still acts by isometries induced by right multiplication in $M \times G$. We can thus also consider iterated Cheeger deformations corresponding to a chain of subgroups $H_{1} \subset \cdots \subset H_{k} \subset G$.

The deformation $g \rightarrow g_{t}$ tends to improve curvature. If the original metric $g$ has non-negative curvature, the deformed metric does also by O'Neill's formula. If $X, Y$ span a 0 -curvature 2-plane of $g$, then, by considering the $G$-components of vectors in $M \times G$, its curvature becomes positive in the metric $g_{t}$ if $\left[P X_{\mathfrak{m}}, P Y_{\mathfrak{m}}\right] \neq 0$, where we have associated to $X \in T_{p} M$ a vector $X_{\mathfrak{m}} \in \mathfrak{m}_{p}$ such that $X_{\mathfrak{m}}^{*}(p)$ is the component of $X$ in the orbit direction. Although this will not be needed in this survey, one finds a detailed study of the basic properties of this deformation in Müter [61]. We mention here only that, if we let $C_{t}=P^{-1} P_{t}$ on $\mathfrak{m}_{p}$ and $C_{t \mid \mathfrak{m}_{p}^{\perp}}=\mathrm{Id}$ be the symmetric endomorphism that expresses $g_{t}$ in terms of $g=g_{0}$, then $\sec _{g_{t}}\left(C_{t}^{-1} X, C_{t}^{-1} Y\right)>0$ for $t>0$ unless $\sec _{g}(X, Y)=0,\left[P X_{\mathfrak{m}}, P Y_{\mathfrak{m}}\right]=0$ and $d \omega_{Z}(X, Y)=0$ for all $Z \in \mathfrak{g}$, where $\omega_{Z}$ is the one form dual to the Killing vector field $Z$. Thus the 0-curvature planes tend to "move" with $C_{t}^{-1}$. Furthermore, 2-planes which are tangent to a totally geodesic flat 2-torus, and which contain a vector orthogonal to the $G$ orbit, remain flat.

As a starting point for finding examples, one considers compact Lie groups $G$ endowed with a biinvariant metric since their curvature satisfies:

$$
\sec _{G}(x, y)=\frac{1}{4}\|[x, y]\|^{2} \geq 0 \quad \text { for } x, y \in \mathfrak{g} \text { orthonormal. }
$$

Combining this fact with O'Neill's formula, we obtain non-negative curvature on every isometric quotient of a compact Lie group. In particular, all homogeneous spaces $G / H$, where $H$ is a closed subgroup of $G$, have metrics with non-negative curvature. Since the identity component of the isometry group of a compact Lie group $G$, endowed with a biinvariant metric, consists of left and right translations, it is natural to generalize the class of homogeneous manifolds to what are called biquotients. Consider $H \subset G \times G$ and define an action of $H$ on $G$ by

$$
h \star g=h_{1} g h_{2}^{-1}, \quad \text { where } \quad h=\left(h_{1}, h_{2}\right) \in H .
$$

The action is free if and only if $h_{1}$ conjugate to $h_{2}$, for $\left(h_{1}, h_{2}\right) \in H$, implies that $h_{1}=h_{2}=e$. If this is the case, the quotient is a manifold, which we denote by $G / / H$ and is called a biquotient. If $H=L \times K \subset G \times G$, we will also write $L \backslash G / K$. Thus we obtain:

THEOREM 2.2. A biinvariant metric on $G$ induces a metric with nonnegative sectional curvature on every homogeneous space $G / H$ and every biquotient $G / / H$. 
The first time where biquotients were considered in geometry, was in [36], where it was shown that an exotic 7-sphere admits non-negative curvature. To describe this example, consider the action of the unit quaternions $\operatorname{Sp}(1)$ on the symplectic group $\operatorname{Sp}(2)$ via:

$$
q \star A=\operatorname{diag}(q, q) A \operatorname{diag}(q, 1)^{-1} \quad q \in \operatorname{Sp}(1), A \in \operatorname{Sp}(2) .
$$

This action is clearly free and we have:

THEOREM 2.3 (Gromoll-Meyer). The non-negatively curved manifold $\mathrm{Sp}(2) / / \mathrm{Sp}(1)$ is homeomorphic, but not diffeomorphic, to $\mathbb{S}^{7}$.

In order to prove this, one observes that $\operatorname{Sp}(2) / / \operatorname{Sp}(1) \operatorname{Sp}(1)=\operatorname{diag}(q, q)$ $A \operatorname{diag}(r, 1)^{-1}, \quad q, r \in \mathrm{Sp}(1)$, is diffeomorphic to $\mathbb{S}^{4}$ since the action of $\operatorname{diag}(q, q)$ on $\operatorname{Sp}(2) / \operatorname{diag}(r, 1)=\mathbb{S}^{7}$ is the Hopf action by $\mathrm{S}^{3}$. Thus $\operatorname{Sp}(2) / /$ $\mathrm{Sp}(1)$ can be considered as an $\mathbb{S}^{3}$ bundle over $\mathbb{S}^{4}$. One then identifies which sphere bundle it represents by using Milnor's description of certain exotic 7 -spheres as $\mathbb{S}^{3}$ bundles over $\mathbb{S}^{4}$. We also point out that in [77], and independently in [55], it was shown that the only exotic sphere which can be written as a biquotient is the Gromoll-Meyer sphere.

Another special class of non-negatively curved examples were constructed in $[\mathbf{1 8}]$ :

THEOREM 2.4 (Cheeger). The connected sum of any two rank one symmetric spaces carries a metric with non-negative sectional curvature.

In [77] it was shown that some of these Cheeger manifolds, but not all, can be viewed as biquotients as well.

The gluing construction used in order to prove Theorem 2.4, was recently significantly generalized to what are called cohomogeneity one manifolds. Recall that if $G$ is a Lie group that acts on a manifold $M$, the cohomogeneity of the action is defined as $\operatorname{cohom}(M, G)=\operatorname{dim} M / G$. Thus an action with $\operatorname{cohom}(M, G)=0$ is an action that is transitive, i.e., the manifold is a homogeneous spaces. Cohomogeneity one manifolds can thus be considered as the next simplest kind of group actions to study. They are also special among all group actions since, as we will see, the manifold can be reconstructed from its isotropy groups. The geometry and topology of homogeneous spaces is fairly well understood by now, whereas this is not yet the case for cohomogeneity one manifolds. One should point out though, that this class of manifolds does not contain the homogeneous spaces as a subset. In fact only very few homogeneous spaces carry a cohomogeneity one action.

Let $G$ be a compact group acting by cohomogeneity one on a compact manifold $M$. Since $M / G$ is one dimensional, it is either a circle $\mathrm{S}^{1}$, or an interval $I$. In the first case all $G$ orbits are principal and $\pi: M \rightarrow M / G=\mathrm{S}^{1}$ is a fiber bundle with fiber a principal orbit $G / H$, and thus the fundamental group is infinite. One also easily sees that such fiber bundles carry a 
$G$ invariant metric with non-negative curvature. In the second more interesting case there are precisely two nonprincipal $G$-orbits corresponding to the endpoints of $I$, and $M$ is decomposed as the union of two tubular neighborhoods of these nonprincipal orbits, with common boundary a principal orbit. Let $\ell_{-}$and $\ell_{+}$be the codimension of the nonprincipal orbits. We have the following existence theorem $[\mathbf{4 2}]$ :

Theorem 2.5 (Grove-Ziller). A compact cohomogeneity one G-manifold with $\ell_{ \pm} \leq 2$ has a $G$-invariant metric with non-negative sectional curvature.

One easily sees that $\ell_{ \pm}>1$ if $M$ is simply connected. Although the assumption on the codimensions seems rather special, it turns out that the class of cohomogeneity one manifolds with $\ell_{ \pm}=2$ is surprisingly rich. An immediate application is:

Corollary 2.6. Each of the 4 oriented diffeomorphism types of $\mathbb{R} \mathbb{P}^{5}$ 's carries a metric with non-negative sectional curvature.

This follows since $\mathbb{S}^{5}$ carries (non-linear) cohomogeneity one actions by $\mathrm{SO}(2) \mathrm{SO}(3)$, discovered by G.Calabi, with codimension 2 singular orbits. They are a special case of the action on Kervaire spheres described below. The involution in $\mathrm{SO}(2)$ acts freely and, using surgery theory, one shows that any one of the exotic $\mathbb{R}^{5}$ 's can be obtained in this fashion, see [60].

In [42] it was also conjectured that Theorem 2.5 holds without any assumption on the codimensions. This turns out to be false. One has [40]:

Theorem 2.7 (Grove-Verdiani-Wilking-Ziller). For each pair $\left(\ell_{-}, \ell_{+}\right)$ with $\left(\ell_{-}, \ell_{+}\right) \neq(2,2)$ and $\ell_{ \pm} \geq 2$ there exist infinitely many cohomogeneity one $G$-manifolds that do not carry a $G$ invariant metric with non-negative sectional curvature.

The most interesting example in this Theorem are the Kervaire spheres, which are the only exotic spheres that can carry a cohomogeneity one action [74]. They are described as a $2 n-1$ dimensional Brieskorn varietiy:

$$
z_{0}^{d}+z_{1}^{2}+\cdots z_{n}^{2}=0, \quad\left|z_{0}\right|^{2}+\cdots\left|z_{n}\right|^{2}=1 .
$$

It carries a cohomogeneity one action by $\mathrm{SO}(2) \mathrm{SO}(\mathrm{n})$ defined by $\left(e^{i \theta}, A\right)$ $\left(z_{0}, \ldots, z_{n}\right)=\left(e^{2 i \theta} z_{0}, e^{i d \theta} A\left(z_{1}, \ldots, z_{n}\right)^{t}\right)$ whose codimensions are $\left(\ell_{-}, \ell_{+}\right)=$ $(2, n-1)$. For $n$ odd and $d$ odd, they are homeomorphic to spheres, and are exotic spheres if $2 n-1 \equiv 1 \bmod 8$. If $n \geq 4, d \geq 3$ one shows that there exists no $G$-invariant metric with non-negative curvature.

To prove Theorem 2.7, one needs to describe the set of all $G$-invariant metrics explicitly. They depend on a finite collection of functions, 6 in the case of the Kervaire spheres, which need to satisfy certain smoothness conditions at the endpoint. For each choice of 2-planes tangent to the principal orbit, one obtains differential inequalities on these functions and their first 
derivatives from the Gauss equations. By a suitable choice of 2-planes one obtains upper and lower bounds on the first derivatives which contradict the smoothness conditions at one of the singular orbits.

We mention that in the case of Ricci curvature one has the positive result that every cohomogeneity one manifold carries an invariant metric with non-negative Ricci curvature, and with positive Ricci curvature if and only if the fundamental group is finite $[\mathbf{4 3}]$.

To discuss the proof of Theorem 2.5 and some of its applications, we first recall the basic structure of cohomogeneity one actions. We will only consider the most interesting case, where $M / G=I$ and let $\pi: M \rightarrow M / G$ be the projection. In order to make the description more explicit, we choose an arbitrary but fixed $G$-invariant Riemannian metric on $M$, normalized so that with the induced metric, $M / G=[-1,1]$. Fix a point $x_{0} \in \pi^{-1}(0)$ and let $c:[-1,1] \rightarrow M$ be a geodesic orthogonal to the orbit through $x_{0}$, and hence to all orbits, and parameterized such that $\pi \circ c=i d_{[-1,1]}$. Let $B_{ \pm}=\pi^{-1}( \pm 1)=G \cdot x_{ \pm}$be the two nonprincipal orbits, where $x_{ \pm}=c( \pm 1)$. It then follows that $c:[2 n-1,2 n+1] \rightarrow M, n \in \mathbb{Z}$ are minimal geodesics between the two nonprincipal orbits $B_{ \pm}$since $G$ acts transitively on the set of all geodesics orthogonal to the orbits. Let $K_{ \pm}=G_{x_{ \pm}}$be the isotropy groups at $x_{ \pm}$and $H=G_{x_{0}}=G_{c(t)},-1<t<1$, the principal isotropy group. By the slice theorem, we have the following description of the tubular neighborhoods $D\left(B_{-}\right)=\pi^{-1}([-1,0])$ and $D\left(B_{+}\right)=\pi^{-1}([0,1])$ of the nonprincipal orbits $B_{ \pm}=G / K_{ \pm}$:

$$
D\left(B_{ \pm}\right)=G \times_{K_{ \pm}} D^{\ell_{ \pm}},
$$

where $D^{\ell_{ \pm}}$is the normal (unit) disk to $B_{ \pm}$at $x_{ \pm}$. Here the action of $K_{ \pm}$on $G \times D^{\ell_{ \pm}}$is given by $k \star(g, p)=\left(g k^{-1}, k p\right)$ where $k$ acts on $D^{\ell_{ \pm}}$via the slice representation. Hence we have the decomposition

$$
M=D\left(B_{-}\right) \cup_{E} D\left(B_{+}\right),
$$

where $E=\pi^{-1}(0)=G \cdot x_{0}=G / H$ is a principal orbit which is canonically identified with the boundaries $\partial D\left(B_{ \pm}\right)=G \times_{K_{ \pm}} \mathbb{S}^{\ell_{ \pm}-1}$, via the maps $G \rightarrow G \times \mathbb{S}^{\ell_{ \pm}-1}, g \rightarrow(g, \mp \dot{c}( \pm 1))$. Note also that $\partial D^{\ell_{ \pm}}=\mathbb{S}^{\ell_{ \pm}-1}=K_{ \pm} / H$ since the boundary of the tubular neighborhoods must be a $G$ orbit and hence $\partial D^{\ell_{ \pm}}$is a $K_{ \pm}$orbit. All in all we see that we can recover $M$ from $G$ and the subgroups $H$ and $K_{ \pm}$. We caution though that the isotropy types, i.e., the conjugacy classes of the isotropy groups $K_{ \pm}$and $H$ do not determine $M$.

An important fact about cohomogeneity one actions is that there is a converse to the above construction. Suppose $G$ is a compact Lie group and $H \subset K_{ \pm} \subset G$ are closed subgroups, which we sometimes denote by $H \subset\left\{K_{-}, K_{+}\right\} \subset G$. Assume furthermore that $K_{ \pm} / H=\mathbb{S}^{\ell_{ \pm}-1}$ are spheres. It is well known that a transitive action of a compact Lie group $K$ on a sphere $\mathbb{S}^{\ell}$ is conjugate to a linear action and is determined by its isotropy group $H \subset K$. We can thus assume that $K_{ \pm}$acts linearly on $\mathbb{S}^{\ell_{ \pm}}$with 
isotropy group $H$ at $p_{ \pm} \in \mathbb{S}^{\ell_{ \pm}-1}$ and define a manifold

$$
M=G \times_{K_{-}} D^{\ell_{-}} \cup_{G / H} G \times_{K_{+}} D^{\ell_{+}},
$$

where we glue the two boundaries by sending $\left[g, p_{-}\right]$to $\left[g, p_{+}\right]$. $G$ acts on $M$ via $g^{*}[g, p]=\left[g^{*} g, p\right]$ on each half and one easily checks that it has isotropy groups $K_{ \pm}$at $[e, 0]$ and $H$ at $\left[e, p_{0}\right]$ and is thus cohomogeneity one.

Theorem 2.5 clearly follows from the following geometric result by gluing two such metrics on the tubular neighborhoods $D\left(B_{ \pm}\right)$along their common boundary $G / H$.

Proposition 2.8. Let $H \subset K \subset G$ be Lie groups with $K / H=\mathbb{S}^{1}=\partial D^{2}$ and fix a biinvariant metric $Q$ on $G$. On the disc bundle $G \times{ }_{K} D^{2}$ there exists a $G$-invariant metric with non-negative sectional curvature, which is a product near the boundary $G \times{ }_{K} \mathbb{S}^{1}=G / H$ with metric on $G / H$ induced by $Q$.

The crucial ingredient in the proof of Proposition 2.8 is the following result about left invariant metrics.

LEMMA 2.9. Let $G$ be a compact Lie group and $\mathfrak{k} \subset \mathfrak{g}$ an abelian subalgebra. Consider the left invariant metric on $G$ whose value at $T_{e} G=\mathfrak{g}$ is given by

$$
Q_{t}=t Q_{\mid \mathfrak{k}}+Q_{\mid \mathfrak{k} \perp}
$$

where $Q$ is a biinvariant metric on $G$. Then $Q_{t}$ has non-negative sectional curvature as long as $t \leq 4 / 3$.

Proof. The curvature formula for a left invariant metric $\langle X, Y\rangle=$ $Q(P X, Y)$ is given by (see e.g. [67]):

$$
\begin{aligned}
\langle R(X, Y) Y, X)\rangle= & \frac{1}{2} Q([P X, Y]+[X, P Y],[X, Y])-\frac{3}{4} Q(P[X, Y],[X, Y]) \\
& +Q\left(B(X, Y), P^{-1} B(X, Y)\right)-Q\left(B(X, X), P^{-1} B(Y, Y)\right),
\end{aligned}
$$

where $B(X, Y)=\frac{1}{2}([X, P Y]-[P X, Y])$.

In our case, let $X=A+R, Y=B+S$ with $A, B \in \mathfrak{m}=\mathfrak{k}^{\perp}$ and $R, S \in \mathfrak{k}$ and hence $P(A+R)=A+t R$. We can now split up the expressions into components in direction of $\mathfrak{m}$ and of $\mathfrak{k}$. A computation shows that the $\mathfrak{m}$ component is given by

$$
\frac{1}{4}\left\|[A, B]_{\mathfrak{m}}+t[X, B]+t[A, Y]\right\|_{Q}^{2} \geq 0
$$

where we have used the bi-invariance of $Q$ and the Jacobi identity to show that $\langle[X, B],[A, Y]\rangle=\langle[X, A],[Y, B]\rangle$. On the other hand, the $\mathfrak{k}$ component is given by

$$
\left\|[A, B]_{\mathfrak{k}}\right\|_{Q}^{2}-\frac{3}{4} t\left\|[A, B]_{\mathfrak{k}}\right\|_{Q}^{2}=\left(1-\frac{3}{4} t\right)\left\|[A, B]_{\mathfrak{k}}\right\|_{Q}^{2},
$$

which is non-negative as long as $t \leq \frac{4}{3}$. 
Proof of Proposition 2.8 We have inclusions $H \subset K \subset G$ with $K / H=\mathbb{S}^{1}$ and define $Q$-orthogonal decompositions $\mathfrak{g}=\mathfrak{k}+\mathfrak{m}$ and $\mathfrak{k}=\mathfrak{h}+\mathfrak{p}$. As usual, we identify the tangent spaces $T_{(H)} K / H \cong \mathfrak{p}$ and $T_{(H)} G / H \cong \mathfrak{p}+\mathfrak{m}$. Since $\mathfrak{p}$ is one dimensional, Lemma 2.9 implies that the left invariant metric on $G$ defined by $Q_{a}=a Q_{\mid \mathfrak{p}}+Q_{\mid \mathfrak{h}+\mathfrak{m}}$ has non-negative curvature as long as $a \leq 4 / 3$. Since $[\mathfrak{p}, \mathfrak{p}]=0$ and $[\mathfrak{h}, \mathfrak{p}] \subset \mathfrak{p}$, the subalgebra $\mathfrak{p}$ is an ideal of $\mathfrak{k}$ and hence Ad $K$ invariant, and thus $Q_{a}$ is right $K$-invariant as well. In addition we choose a metric $g_{f}=d t^{2}+f(t)^{2} d \theta^{2}$ on $D^{2}$ which is clearly invariant under the action of $K$ on $D^{2}$ and has non-negative curvature if $f$ is concave. The product metric $Q_{a}+g_{f}$ on $G \times D^{2}$ thus induces a non-negatively curved metric $g_{a, f}$ on the homogeneous disk bundle $G \times_{K} D^{2}$. We now claim that given $1<a \leq 4 / 3$, we can choose $f$ such that $g_{a, f}$ is a product near the boundary with metric on $G / H$ induced by $Q$. To see this, consider the Riemannian submersion $G \times(K / H) \rightarrow G \times{ }_{K} K / H \cong G / H$ where we endow $K / H=\mathbb{S}^{1}$ with the metric of a circle of radius $f(t)$. The induced metric on $G / H$ is the metric $g_{a, f}$ restricted to the boundary of a tube of radius $t$. We compute this metric as in the case of a Cheeger deformation (2.1). If $2 \pi s_{0}$ is the length of the circle $K / H$ in the metric $Q_{\mid \mathfrak{p}}$, the metric on $K / H$ is given by $\left(f / s_{0}\right)^{2} Q$ and it follows that the metric on $G / H$ is given by $Q$ on $\mathfrak{m}$ and by $\frac{a}{1+a\left(f / s_{0}\right)^{-2}} Q=\frac{f^{2} a}{f^{2}+a s_{0}^{2}} Q$ on $\mathfrak{p}$. Hence we obtain the desired metric by choosing a concave function $f$ and a $t_{0}$ such that $f^{2}(t)=\frac{a s_{0}^{2}}{a-1}$, for $t \geq t_{0}$.

Remark. We can view this construction as a "scaling up, scaling down" procedure. The natural metric on $G \times{ }_{K} D$ induced by a biinvariant metric $Q$ on $G$ shrinks the metric on the boundary $G / H$ in the direction of $K / H$, as in the case of a Cheeger deformation. This needs to be compensated by scaling the metric $Q$ up in the direction of $\mathfrak{p}$ in order to recover the metric $Q$ on $G / H$. This explains the difficulty of proving Proposition 2.8 for $\ell>2$ since left invariant metrics as in Lemma 2.9 in general have some sectional curvature positive when $t>1$. In fact we believe:

Problem 1. Let $G$ be a compact simple Lie group and $K$ a non-abelian subgroup. Show that a metric $Q_{t}$ as in Lemma 2.9 has some negative sectional curvatures for any $t>1$.

Nevertheless, it is possible that there are other special homogeneous disk bundles for which Proposition 2.8 holds with codimension $\ell>2$.

As was observed by B. Wilking, Theorem 2.5 can be generalized to the situation where the homogeneous orbits are replaced by biquotients. In other words, if $K_{ \pm} \subset G \times G$ acts freely on $G$ and $H \subset K_{ \pm}$with $K_{ \pm} / H=\mathbb{S}^{1}$, then the resulting manifold carries a metric with non-negative curvature. This follows by applying Theorem 2.5 to the cohomogeneity one manifold $H \subset\left\{K_{-}, K_{+}\right\} \subset G \times G$ and then dividing by $\Delta G \subset G \times G$ on the left, which acts freely by assumption. 
We now apply this result to some concrete cohomogeneity one manifolds in order to prove:

Theorem 2.10 (Grove-Ziller). Every principal $\mathrm{SO}(k)$ bundle P over $\mathbb{S}^{4}$ carries a cohomogeneity one action by $\mathrm{SO}(3) \times \mathrm{SO}(k)$ with codimension two principal orbits and hence an invariant metric with non-negative curvature.

Thus, by O'Neill's formula, every associated bundle $P \times_{\mathrm{SO}(k)} X$ with $X$ a non-negatively curved manifold on which $\mathrm{SO}(k)$ acts by isometries, also carries a non-negatively curved metric.

Corollary 2.11. Every sphere bundle over $\mathbb{S}^{4}$ carries a metric with non-negative sectional curvature.

Of particular interest are $\mathbb{S}^{3}$ bundles over $\mathbb{S}^{4}$ since Milnor discovered the first exotic spheres among these manifolds. It implies:

Corollary 2.12. Of the 14 (unoriented) exotic 7-spheres, 10 carry a metric with non-negative curvature.

The group of exotic spheres, under the group operation of connected sums, is isomorphic to $\mathbb{Z}_{28}$, but a change of orientation corresponds to taking an inverse. It is not known whether the remaining 4 exotic spheres carry non-negative curvature metrics as well.

Proof of Theorem 2.10: Let the cohomogeneity one manifold $P_{r, s}$ be given by the isotropy groups:

$$
H=\triangle Q \subset\left\{\left(e^{i r \theta}, e^{i \theta}\right) \cdot H,\left(e^{j s \theta}, e^{j \theta} \cdot H\right\} \subset \mathrm{S}^{3} \times \mathrm{S}^{3},\right.
$$

where $\triangle Q=\{ \pm(1,1), \pm(i, i), \pm(j, j), \pm(k, k)$,$\} is the quaternion group and$ $e^{i r \theta}=\cos (r \theta)+i \sin (r \theta)$ is an embedding of a circle into the unit quaternions $\mathrm{S}^{3}$. In order for $H$ to be a subgroup of $K_{ \pm}$, we need to assume that $r, s \equiv 1 \bmod 4$. We then have $K_{ \pm} / H=\mathrm{S}^{1}$ and thus Theorem 2.5 implies that $P_{r, s}$ carries an $\mathrm{S}^{3} \times \mathrm{S}^{3}$ invariant metric with non-negative curvature. The subgroup $\mathrm{S}^{3}=\mathrm{S}^{3} \times\{e\} \subset \mathrm{S}^{3} \times \mathrm{S}^{3}$ acts freely on $P_{r, s}$ since its isotropy groups are the intersection of $\mathrm{S}^{3} \times\{e\}$ with $K_{ \pm}$and $H$, which by construction are trivial. We now claim that $P_{r, s} / \mathrm{S}^{3}$ is $\mathbb{S}^{4}$. To see this, observe that the second $\mathrm{S}^{3}$ factor induces a cohomogeneity one action with group diagram $Q \subset\left\{e^{i \theta} \cdot Q, e^{j \theta} \cdot Q\right\} \subset \mathrm{S}^{3}$ on the quotient. The element $-1 \in \mathrm{S}^{3}$ acts trivially and the effective version of the action has isotropy groups $\mathbb{Z}_{2} \times \mathbb{Z}_{2} \subset\{\mathrm{S}(\mathrm{O}(2) \mathrm{O}(1)), \mathrm{S}(\mathrm{O}(1) \mathrm{O}(2))\} \subset \mathrm{SO}(3)$. But there is a well known linear action by $\mathrm{SO}(3)$ on $\mathbb{S}^{4}$ given by conjugation on the set of $3 \times 3$ symmetric real matrices with trace 0 . Since every matrix is conjugate to a diagonal one, it follows that the two singular orbits are given by symmetric matrices with two equal eigenvalues, positive for one and negative for the other, and the principal orbits by matrices with 3 distinct eigenvalues. One now easily 
checks that the isotropy groups are the same as for the above action and hence $P_{r, s} / \mathrm{S}^{3}$ is equivariantly diffeomorphic to $\mathbb{S}^{4}$.

Thus $P_{r, s}$ can be viewed as an $\mathrm{S}^{3}$ principal bundle over $\mathbb{S}^{4}$. These are classified by an integer $k$, namely the Euler class of the bundle evaluated on a fixed orientation class of $\mathbb{S}^{4}$. To recognize which bundle it is, one observes that the Gysin sequence implies $H^{4}(E, \mathbb{Z})=\mathbb{Z}_{|k|}$ for such a bundle. For a cohomogeneity one manifold one can compute the cohomology groups by using Meyer-Vietoris on the decomposition into the disk bundles $D\left(B_{ \pm}\right)$. The disk bundles are homotopy equivalent to $G / K_{ \pm}$and their intersection to $G / H$. Using well known methods for computing the cohomology groups of homogeneous spaces one shows that $H^{4}\left(P_{r, s}, \mathbb{Z}\right)$ is a cyclic group of order $\left(r^{2}-s^{2}\right) / 8$. But for $r, s \equiv 1 \bmod 4$ the values of $\left(r^{2}-s^{2}\right) / 8$ can take on any integer. Thus every $\mathrm{S}^{3}$ principal bundle over $\mathbb{S}^{4}$ is of the form $P_{r, s}$ for some $r, s$. Since every $\mathrm{SO}(3)$ principal bundle over $\mathbb{S}^{4}$ is spin, i.e., has a lift to an $\mathrm{S}^{3}$ principal bundle, this implies Theorem 2.10 for $k=3$. The case of $k=4$ one obtains by repeating the above argument for $G=\mathrm{S}^{3} \times \mathrm{S}^{3} \times \mathrm{S}^{3}$ with $\mathrm{K}_{ \pm}$again one dimensional and identity component of say $K_{-}$equal to $\left(e^{i r_{1} \theta}, e^{i r_{2} \theta}, e^{i \theta}\right)$ with $r_{i} \in \mathbb{Z}$. For principal bundles $P$ over $\mathbb{S}^{4}$ with $k>4$ it is well known that their structure group reduces to $\mathrm{SO}(4)$. Thus there exists an $\mathrm{SO}(4)$-principal bundle $P^{\prime}$ with $P=P^{\prime} \times{ }_{\mathrm{SO}(4)} \mathrm{SO}(k)$ on which $\mathrm{SO}(k)$ acts on the right. Hence the lift of $\mathrm{SO}(3)$ to $P^{\prime}$ also lifts to $P$ and commutes with $\mathrm{SO}(k)$.

We finally indicate how the proof of Cheeger's Theorem 2.4 fits into the above framework. Of the connected sums considered in his theorem, only $\mathbb{C} \mathbb{P}^{n} \#-\mathbb{C} \mathbb{P}^{n}$ admits a cohomogeneity one action. But a similar idea as in the proof of Proposition 2.8 applies to all cases. A rank one projective space $M^{n}$ with a small ball removed, is diffeomorphic to the disk bundle of the canonical line bundle over the projective space of one dimension lower. This bundle is a homogeneous disk bundle with boundary diffeomorphic to a sphere. One now uses the same "scaling up, scaling down" method as in the proof of Proposition 2.8 to show that these disk bundles have a metric with non-negative curvature which is a product near the boundary and has constant curvature one on the boundary. One can then glue together any two rank one projective spaces along this boundary.

The methods described in the proof of Theorem 2.10 can also be applied to other 4-manifolds as base [44]:

TheOREM 2.13 (Grove-Ziller). Every principal $\mathrm{SO}(k)$ bundle $P$ over $\mathbb{C P}^{2}$ which is not spin, i.e., $w_{2}(P) \neq 0$, carries a cohomogeneity one action with codimension two principal orbits and hence an invariant metric with nonnegative curvature. Thus, so does every associated sphere bundle.

To prove this, one uses the linear cohomogeneity one action on $\mathbb{C P}^{2}$ given by $\mathrm{SO}(3) \subset \mathrm{SU}(3)$, which one easily verifies has group diagram $\mathbb{Z}_{2} \subset$ $\{\mathrm{S}(\mathrm{O}(2) \mathrm{O}(1)), \mathrm{SO}(2)\} \subset \mathrm{SO}(3)$ and constructs a group diagram with $G=\mathrm{S}^{3} \times \mathrm{S}^{3}$ as above, but with $H=\{( \pm 1, \pm 1),( \pm i, \pm i)\}$. The topological 
considerations needed to identify what bundle the cohomogeneity one manifold represents, are significantly more difficult. It also raises the following general question, which the above examples show is important in the context of cohomogeneity one manifolds.

Given a principal $L$ bundle $P \rightarrow M$ over a $G$-manifold $M$. When does the action of $G$ on $M$ have a commuting lift, i.e., a lift to an action of $G$, or possibly a cover of $G$, on the total space $P$, such that the lift commutes with $L$.

This problem has been studied extensively. However, apart from the general result that every action of a semi simple group admits a commuting lift to the total space of every principal circle or more generally torus bundle [63], the results seem to be difficult to apply in concrete cases. For a cohomogeneity one manifold $M_{G}$ with isotropy groups $H \subset\left\{K_{-}, K_{+}\right\} \subset G$, one has a natural description of the lifts to an $L$-principal bundle over $M_{G}$ in terms of the isotropy groups. Simply choose embeddings of $K_{ \pm}$into $L \times G$ such that they agree on $H$ and are given in the second component by the original embeddings into $G$. The action by $L \times\{e\}$ is then clearly free, and the quotient is $M_{G}$ since the induced $G$ action has the same isotropy groups. As long as one allows the action of $G$ on $M_{G}$ to be ineffective, all lifts are described in this fashion. The difficulty is then to decide what the isomorphism type of this $L$-principal bundle is.

Theorem 2.10 and Theorem 2.13 can be restated as saying that the linear actions of $\mathrm{SO}(3)$ on $\mathbb{S}^{4}$ and $\mathbb{C P}^{2}$ have a commuting lift to every principal $\mathrm{SO}(k)$ bundle, respectively principal $\mathrm{SO}(k)$ bundle which is not spin. In [44] one finds a classification of which cohomogeneity one actions on simply connected 4-manifolds $M^{4}$ have a commuting lift to a given principal $\mathrm{SO}(k)$ bundle over $M^{4}$. In particular, it is shown that in the spin case the action of $\mathrm{SO}(3)$ on $\mathbb{C P}^{2}$ only lifts to half of all $\mathrm{SO}(3)$ principal bundles. This shows the limitations of our principal bundle method which finds metrics on their total space with $\mathrm{sec} \geq 0$.

A particularly interesting case of the above Problem are $\mathrm{SO}(k)$ principal bundles over $\mathbb{S}^{k}$ since cohomogeneity one actions on spheres are numerous and have been classified in [52].

Problem 2. Which cohomogeneity one actions on $\mathbb{S}^{n}$ admit a commuting lift to a given $\mathrm{SO}(k)$ principal bundle over $\mathbb{S}^{n}$ ?

An answer to this question could potentially produce further sphere bundles over spheres, and hence possibly higher dimensional exotic spheres, which carry metrics with non-negative curvature.

In light of the existence Theorem 2.5 and the non-existence Theorem 2.7, it is natural to pose the following somewhat vague but important:

PROBLem 3. How large is the class of cohomogeneity one manifolds that admit an invariant metric with non-negative curvature? 
Are there other obstructions, and how strong are the obstructions developed in the proof of Theorem 2.7? As far as existence is concerned, one would need to understand how to put non-negative curvature on cohomogeneity one manifolds without making the middle totally geodesic. In [69] Schwachhöfer showed that for the adjoint action of $\mathrm{SU}(3)$ on $\mathbb{S}^{7} \subset \mathfrak{s u}(3)$ there exist no invariant metric with non-negative curvature such that the middle is totally geodesic (for any homogeneous metric on the principal orbit!). But there of course exists an invariant metric with positive curvature.

We end this section with the following natural problem. Many examples are obtained by taking a quotient of a compact Lie group, equipped with a left invariant metric with $\mathrm{sec} \geq 0$, by a group acting by isometries. It thus seems to be important to know what all such metrics look like.

Problem 4. Classify all left invariant metrics with non-negative sectional curvature on compact Lie groups.

Surprisingly, the only examples known so far are obtained by combining the following: Cheeger deformations of a biinvariant metric along a subgroup $K$, i.e. the metric on $G=G \times_{K} K$ induced by $Q+\frac{1}{t} Q$. If the subgroup is 3 -dimensional, we can more generally consider the metric on $G$ induced by $Q+\frac{1}{t} g$ where $g$ is a left invariant metric on $K$ with positive curvature. Finally, we can scale a biinvariant metric up in the direction of an abelian subalgebra as in Lemma 2.9. The only Lie groups where a complete answer is known, are $\mathrm{SU}(2)$ and $\mathrm{U}(2)$, see [15], and with partial results for $\mathrm{SO}(4)$, [51]. In the latter paper it was also shown, as another application of Cheeger deformations, that every left invariant non-negatively curved metric $g$ on a compact Lie group $G$ can be connected by an "inverse linear" path of non-negatively curved left invariant metrics to a fixed biinvariant metric $Q$. Indeed, in the Cheeger deformation (2.1) applied to the right action of $G$ on itself, we can let $t \rightarrow \infty$ and then the rescaled metric $t g_{t} \rightarrow Q$ since the eigenvalues of $t g_{t}$ in terms of $Q$ are $\frac{t \lambda_{i}}{1+t \lambda_{i}}$. Thus the main interest lies in deciding what derivatives are allowed at $Q$ for an inverse linear path of left invariant metrics with sec $\geq 0$. This approach is discussed in detail in [51].

\section{Topology of non-negatively curved manifolds}

For the following we assume that our manifolds are compact and simply connected. Recall the Bott conjecture which states that a non-negatively curved manifold is elliptic. Even rationally elliptic, i.e., where the condition on the Betti numbers of the loop space is only assumed for rational coefficients, already has strong consequences. By Sullivan's theory of minimal models in rational homotopy theory, rationally elliptic is equivalent to saying that there are only finitely many homotopy groups which are not finite. Rationally elliptic implies that the sum of the Betti number of $M^{n}$ is at most $2^{n}$, which is the optimal upper bound in Gromov's Betti number theorem. Furthermore, the Euler characteristic is non-negative (one half of 
the Hopf conjecture), and positive if and only if the odd Betti numbers are 0 . Thus it is natural to conjecture that an even dimensional manifold with positive curvature has vanishing odd Betti numbers. See [37] where geometric consequences for rationally elliptic as well as for the remaining class of simply connected so-called rationally hyperbolic manifolds were first discussed in detail.

In dimension four, rationally elliptic manifolds are homeomorphic to one of the known examples with non-negative curvature, i.e., one of $\mathbb{S}^{4}, \mathbb{C P}^{2}$, $\mathbb{S}^{2} \times \mathbb{S}^{2}$ or $\mathbb{C P}^{2} \# \pm \mathbb{C P}^{2}$. It is natural to conjecture that a non-negatively curved manifold is indeed diffeomorphic to one of these, and that only the first two can admit positive curvature. In [65] it was shown that an elliptic 5 -manifold is diffeomorphic to one of the known examples with non-negative curvature, i.e., one of $\mathbb{S}^{5}, \mathrm{SU}(3) / \mathrm{SO}(3), \mathbb{S}^{3} \times \mathbb{S}^{2}$ or the non-trivial $\mathbb{S}^{3}$ bundle over $\mathbb{S}^{2}$. Thus the Bott conjecture in dimension 5 states that a non-negatively curved manifold is diffeomorphic to one of these models, and it is natural to conjecture that only the first admits positive curvature.

We remark that in dimension two a non-negatively curved manifold is diffeomorphic to $\mathbb{S}^{2}$ by Gauss-Bonnet and in dimension three to $\mathbb{S}^{3}$ by Hamilton's theorem $[\mathbf{4 6}]$.

We now describe some topological properties of the known examples with non-negative curvature. A homogeneous manifold $M$ is 2-connected iff $M=G / H$ with $G$ and $H$ semisimple and hence there are only finitely many such manifolds in each dimension. If it is not 2-connected, $M$ is a torus bundle over a 2-connected one. In [77] it was shown that both statements also hold for biquotients.

On the other hand, the class of biquotient manifolds is significantly larger than the class of homogeneous spaces. For example, in [78] it was shown that there exist infinitely many 6-dimensional biquotients of the form $\left(S^{3}\right)^{3} / /\left(S^{1}\right)^{3}$ with non-isomorphic rational cohomology rings. On the other hand, compact simply connected homogeneous spaces in dimension 6 are either diffeomorphic to a product of rank one symmetric spaces or to the Wallach manifold $\mathrm{SU}(3) / T^{2}$.

The class of cohomogeneity one manifolds, including associated bundles and quotients, is again much larger than both. Indeed, there are infinitely many homotopy types of 2-connected cohomogeneity one manifolds, starting in dimension 7 , since all $\mathbb{S}^{3}$ bundles over $\mathbb{S}^{4}$ admit non-negative curvature. In Section 6 one also finds an infinite family of 7 dimensional cohomogeneity one manifolds, depending on 4 arbitrary integers, which are 2-connected and have singular orbits of codimension two.

In $[\mathbf{2 4}]$ it was shown that there are infinitely many non-negatively curved manifolds lying in distinct cobordism classes. One starts with one of the principal $\mathrm{SO}(3)$ bundles $P$ over $\mathbb{S}^{4}$ in Theorem 2.10 and considers the associated bundle $P \times_{\mathrm{SO}(3)} \mathbb{C P}^{2}$, where $\mathrm{SO}(3) \subset \mathrm{SU}(3)$ acts linearly on $\mathbb{C P}^{2}$. It clearly has non-negative curvature and a computation of the Pontryagin classes shows that they have distinct Pontryagin numbers and hence lie in 
different cobordism groups. On the other hand, this is not possible for homogeneous spaces and biquotients since circle bundles are the boundary of the associated disk bundle and hence have vanishing Pontryagin numbers.

In [49] C. Hoelscher classified compact simply connected cohomogeneity one manifolds of dimension at most seven. In dimension 4 this was done in [64] (dimension 2 and 3 being trivial). In dimension 5, 6 and 7 there are many cohomogeneity actions with singular orbits of codimension 2 . In dimension 7 there are also some new families whose codimensions are not both two, where it is not known if they carry invariant metrics with nonnegative curvature. On the other hand, one also has the exotic Kervaire spheres in dimension 7 , which by Theorem 2.7 does not admit an invariant metric with non-negative curvature.

\section{Non-compact examples with non-negative curvature}

For non-compact manifolds one has the well known Soul Theorem [19]:

THEOREM 3.1 (Cheeger-Gromoll). If $M^{n}$ is a non-compact manifold with a complete metric with $\mathrm{sec} \geq 0$, then there exists a totally geodesic compact submanifold $S^{k}$ such that $M^{n}$ is diffeomorphic to the normal bundle of $S^{k}$.

The submanifold $S^{k}$ is called the soul of $M^{n}$. A major open problem in this part of the subject is hence:

Problem 5. What vector bundles over compact manifolds with nonnegative curvature admit a complete metric with non-negative sectional curvature?

This is particularly interesting for vector bundles over spheres. Any homogeneous vector bundle $G \times_{K} V$, where $K$ acts orthogonally on a vector space $V$, clearly admits such a metric by O'Neill's formula. Thus $T \mathbb{S}^{n}=$ $\mathrm{SO}(n+1) \times{ }_{\mathrm{SO}(n)} \mathbb{R}^{n}$ also does. Every vector bundle over $\mathbb{S}^{n}, n=1,2,3$ is a homogeneous vector bundle and hence carries non-negative curvature. In [68] Rigas showed that every vector bundle of $\mathbb{S}^{n}$ is stably, i.e., after taking the connected sum with a trivial bundle of sufficiently large dimension, a homogeneous vector bundle and hence carries non-negative curvature.

As a consequence of Theorem 2.10 and Theorem 2.13 one obtains nonnegative curvature on the vector bundles $P \times_{\mathrm{SO}(k)} \mathbb{R}^{k}$ associated to the principal bundles $P$ :

Corollary 3.2 (Grove-Ziller). Every vector bundle over $\mathbb{S}^{4}$, and every vector bundle over $\mathbb{C P}^{2}$ which is not spin, carries a complete metric with non-negative sectional curvature.

This class of vector bundles is quite large since they are classified by one arbitrary integer when the fiber dimension is three and by 2 if the fiber dimension is four. 
As far as vector bundles are concerned over the remaining known 4manifolds with non-negative curvature, i.e. $\mathbb{S}^{2} \times \mathbb{S}^{2}$ and $\mathbb{C P}^{2} \# \pm \mathbb{C P}^{2}$, most of them also admit non-negative curvature since their structure group reduces to a torus and circle bundles over these manifolds are known to admit nonnegative curvature, see $[\mathbf{8 8}, \mathbf{7 7}]$. For vector bundles over $\mathbb{S}^{n}, n>4$, one knows that all vector bundles over $\mathbb{S}^{5}$, and most of the vector bundles over $\mathbb{S}^{7}$, admit non-negative curvature $[\mathbf{4 2}]$. But in both cases there are only finitely many such bundles.

If the base does not have finite fundamental group, there are obstructions to the existence of complete metrics with non-negative curvature due to Özaydin-Walschap [62], in the case where the soul is flat, and BelegradekKapovitch $[\mathbf{6 , 7}]$ in general. The simplest examples are:

THEOREM 3.3. Every orientable vector bundle over $\mathrm{T}^{2}$ or $\mathbb{S}^{3} \times \mathbb{S}^{1}$ with non-negative curvature is trivial.

In $[6,7]$ the authors give many more examples of vector bundles over $C \times T^{k}$ with $\mathrm{C}$ compact and simply connected and $k \geq 1$ which do not admit non-negative curvature. For example, if $k \geq 4$, there exist infinitely many vector bundles over $C \times T^{k}$ of every fixed rank at least two, whose total space do not admit any complete metric with non-negative curvature. No obstructions are known when the base is simply connected.

Although it is known that for a given metric on $M^{n}$ any two souls are isometric, $M$ can have two distinct non-negatively curved metrics with souls that are not even homeomorphic. In fact Belegradek [5] proved:

THEOREM 3.4 (Belegradek). For each $n \geq 5$, there exist infinitely many complete Riemannian metrics on $\mathbb{S}^{3} \times \mathbb{S}^{4} \times \mathbb{R}^{n}$ with sec $\geq 0$ and pairwise non-homeomorphic souls.

To prove this, consider the principal SO(3) bundle $P_{k} \rightarrow \mathbb{S}^{4}$ corresponding to $k \in \pi_{3}(\mathrm{SO}(3)) \cong \mathbb{Z}$ and let $E_{k}^{n}=P_{k} \times{ }_{\mathrm{SO}(3)} \mathbb{R}^{n}$ and $S_{k}^{n}=P_{k} \times_{\mathrm{SO}(3)} \mathbb{S}^{n-1}$ be the associated vector bundle and sphere bundle coming from the standard inclusion $\mathrm{SO}(3) \subset \mathrm{SO}(n)$. Then the bundle $\Delta^{*}\left(S_{k}^{4} \times E_{-k}^{n}\right)$, where $\Delta: \mathbb{S}^{4} \rightarrow \mathbb{S}^{4} \times \mathbb{S}^{4}$ is the diagonal embedding, can be regarded as a bundle over $\mathbb{S}^{4}$ associated to the principal $\mathrm{SO}(3) \times \mathrm{SO}(3)$ bundle $\Delta^{*}\left(P_{k}^{4} \times P_{-k}^{n}\right)$, which by Theorem 2.10 carries an invariant metric with non-negative curvature. On the other hand, it can also be regarded as an n-dimensional vector bundle over $S_{k}^{4}$ and its soul, since it is an associated vector bundle, is equal to the 0 -section $S_{k}^{4}$. Now one uses surgery theory to show that for $k \equiv k^{\prime} \bmod 12$ and $n \geq 5$, the manifold $\Delta^{*}\left(S_{k}^{4} \times E_{-k}^{n}\right)$ is diffeomorphic to $\mathbb{S}^{3} \times \mathbb{S}^{4} \times \mathbb{R}^{n}$ and a computation of the Pontryagin classes shows that $S_{k}^{4}$ is homeomorphic to $S_{k^{\prime}}^{4}$ if and only if $k= \pm k^{\prime}$.

See [5] and [53] for further examples of this type. 


\section{Known examples with positive curvature}

Known examples with positive curvature are surprisingly rare. What is even more surprising is that they are all obtained as quotients of a compact Lie group equipped with a biinvariant or a Cheeger deformation of a biinvariant metric divided by a group of isometries. One may view the following theorem as an explanation of why it is so difficult to find new examples [85].

THEOREM 4.1 (Wilking). If $M^{n}$ admits a positively curved metric with an isometric action of cohomogeneity $k \geq 1$ with $n>18(k+1)^{2}$, then $M$ is homotopy equivalent to a rank one symmetric space.

Thus, for any new examples, the larger the dimension, the bigger the cohomogeneity. This may increase the difficulty of computing its curvature tensor and estimating the sectional curvature. In fact, known examples exist only in low dimensions. They consist of certain homogeneous spaces in dimensions 6,7,12,13 and 24 due to Berger [10], Wallach [86], and AloffWallach [2], and of biquotients in dimensions 6,7 and 13 due to Eschenburg $[\mathbf{2 7}, \mathbf{2 8}]$ and Bazaikin $[\mathbf{3}]$. The purpose of this section is to discuss these examples.

The main ingredient for all known examples is the following Cheeger deformation of a fixed biinvariant metric $Q$ on $G$, of a type we already considered in Lemma 2.9. Let $K \subset G$ be a closed Lie subgroup with Lie algebras $\mathfrak{k} \subset \mathfrak{g}$ and $\mathfrak{g}=\mathfrak{k}+\mathfrak{m}$ a $Q$-orthogonal decomposition. Recall that $(G, K)$ is a symmetric pair if $K$ is, up to components, the fixed point set of an involutive automorphism. For our purposes, the property that $[\mathfrak{m}, \mathfrak{m}] \subset \mathfrak{k}$ is all that is needed, and is equivalent to being a symmetric pair if $G / K$ is simply connected.

For the biinvariant metric $Q$ a 0 -curvature 2-plane is characterized by $[X, Y]=0$. The following deformation thus decreases the set of 0 -curvature 2-planes $[\mathbf{2 8}]$.

LEMma 4.2 (Eschenburg). Let $Q_{t}$ be a left invariant metric on $G$ defined by $Q_{t}=t Q_{\mid \mathfrak{k}}+Q_{\mid \mathfrak{m}}$. Then $\sec _{Q_{t}} \geq 0$ as long as $t \leq 1$. If we assume in addition that $(G, K)$ is a symmetric pair, $X, Y$ span a 0-curvature 2-plane of $g_{t}$, for $t<1$, if and only if $[X, Y]=\left[X_{\mathfrak{k}}, Y_{\mathfrak{k}}\right]=\left[X_{\mathfrak{m}}, Y_{\mathfrak{m}}\right]=0$.

Proof. The metric $Q_{t}$ can be viewed as a Cheeger deformation as in (2.1) with respect to the right action of $K$ on $G$ and hence has non-negative curvature for $t \leq 1$. As we saw, the metric $Q+\frac{1}{s} Q$ on $G \times K$ induces a metric of the form $Q_{t}$ with $t=\frac{1}{s+1}<1$ and the horizontal lift of $X=X_{\mathfrak{k}}+X_{\mathfrak{m}} \in$ $\mathfrak{k}+\mathfrak{m}=\mathfrak{g}$ is equal to $\bar{X}=\left(X_{\mathfrak{m}}+\frac{1}{1+s} X_{\mathfrak{k}},-\frac{s}{1+s} X_{\mathfrak{k}}\right) \in \mathfrak{g}+\mathfrak{k}$. Since the metric on $G \times K$ is biinvariant, a horizontal 2-plane spanned by $\bar{X}, \bar{Y}$ has 0 curvature if and only if $[\bar{X}, \bar{Y}]=0$. Since the O'Neill tensor is also given in terms of Lie brackets, the same is true for the 2-plane spanned by $X, Y \in \mathfrak{g}$. If $G / K$ 
is a symmetric pair, we have $[\mathfrak{m}, \mathfrak{m}] \subset \mathfrak{k}$, which, together with $[\mathfrak{k}, \mathfrak{k}] \subset \mathfrak{k}$ and $[\mathfrak{k}, \mathfrak{m}] \subset \mathfrak{m}$, easily implies the claim.

Given Lie subgroups $H \subset K \subset G$, we define a homogeneous fibration

$$
K / H \longrightarrow G / H \longrightarrow G / K \quad: \quad g H \rightarrow g K .
$$

Using the $Q$-orthogonal decompositions $\mathfrak{g}=\mathfrak{k}+\mathfrak{m}$ and $\mathfrak{k}=\mathfrak{h}+\mathfrak{p}$, we can identify the tangent spaces $\mathfrak{p} \cong T_{(H)} K / H, \mathfrak{m} \cong T_{(K)} G / K$ and $\mathfrak{p}+\mathfrak{m} \cong$ $\left.T_{(H}\right) G / H$. In terms of these identifications, we define a one parameter family of homogeneous metrics on $G / H$ by

$$
g_{t}=t Q_{\mid \mathfrak{p}}+Q_{\mid \mathfrak{m}}
$$

which scales the fibers of the homogeneous fibrations by $t$. Notice that they can also be viewed as a Cheeger deformation of the metric $Q$ on $G / H$ in direction of the left action of $K$ on $G / H$. It is natural to ask, if one has such a metric with positive curvature on the base and on the fiber, when does $g_{t}$ have positive curvature. A partial answer to this question is given by $[\mathbf{8 6}]$ :

Proposition 4.3 (Wallach). Given a homogeneous fibration as above, assume that:

(a) The base $(G, K)$ is a compact symmetric pair of rank one.

(b) The metric on the fiber $K / H$ induced by $Q$ has positive curvature.

(c) For any non-zero vectors $X \in \mathfrak{p}$ and $Y \in \mathfrak{m}$ we have $[X, Y] \neq 0$.

Then the metric $g_{t}$ with $t<1$ has positive sectional curvature.

PROOF. The interpretation as a Cheeger deformation implies that $\sec _{g_{t}} \geq$ 0 for $t \leq 1$. If we define the left invariant metric $Q_{t}$ on $G$ by $Q_{t}=t Q_{\mid \mathfrak{k}}+Q_{\mid \mathfrak{m}}$, the projection $G \rightarrow G / H$ is a Riemannian submersion with respect to the metrics $Q_{t}$ and $g_{t}$. Thus, if $X, Y \in \mathfrak{p}+\mathfrak{m} \cong T_{H} G / H$ span a 0 -curvature 2-plane of $g_{t}$, they span a 0-curvature 2-plane of $Q_{t}$ as well and hence $[X, Y]=\left[X_{\mathfrak{k}}, Y_{\mathfrak{k}}\right]=\left[X_{\mathfrak{m}}, Y_{\mathfrak{m}}\right]=0$ by Lemma 4.2 . The vectors $X_{\mathfrak{k}}, Y_{\mathfrak{k}} \in \mathfrak{p}$ can be viewed as spanning a 2-plane of the fiber $K / H$ and since it is assumed to have positive curvature, $X_{\mathfrak{k}}, Y_{\mathfrak{k}}$ must be linearly dependent. Similarly, since the base has positive curvature, $X_{\mathfrak{m}}, Y_{\mathfrak{m}}$ are linearly dependent. Hence we can find a new basis of this plane with $X \in \mathfrak{p}$ and $Y \in \mathfrak{m}$. But now condition (c) implies that $[X, Y]=0$ is impossible and thus $\sec _{g_{t}}>0$.

The condition that $[X, Y] \neq 0$ is equivalent to the positivity of the curvature of the 2-plane spanned by $X, Y$, i.e. the vertizontal sectional curvatures. This condition is the fatness condition we will discuss in Section 6 .

\section{Homogeneous examples with positive curvature}

Homogeneous spaces which admit a homogeneous metric with positive curvature have been classified by Wallach in even dimensions [86] and by 
Bérard-Bergery in odd dimensions [9]. We now describe these examples, leaving out the compact rank one symmetric spaces as well known. In all cases except for one, we will show that they indeed carry a metric with positive curvature as a consequence of Proposition 4.3.

1) The first class of examples are the homogeneous flag manifolds due to Wallach: $W^{6}=\mathrm{SU}(3) / \mathrm{T}^{2}, W^{12}=\mathrm{Sp}(3) / \mathrm{Sp}(1)^{3}$ and $W^{24}=\mathrm{F}_{4} / \operatorname{Spin}(8)$. They are the total space of the following homogeneous fibrations:

$$
\begin{gathered}
\mathbb{S}^{2} \rightarrow \mathrm{SU}(3) / \mathrm{T}^{2} \rightarrow \mathbb{C P}^{2}, \\
\mathbb{S}^{4} \rightarrow \mathrm{Sp}(3) / \mathrm{Sp}(1)^{3} \rightarrow \mathbb{H} \mathbb{P}^{2}, \\
\mathbb{S}^{8} \rightarrow \mathrm{F}_{4} / \mathrm{Spin}(8) \rightarrow \mathrm{CaP}^{2} .
\end{gathered}
$$

We now show that $W^{6}=\mathrm{SU}(3) / \mathrm{T}^{2}$ has positive curvature, the other cases being similar. Consider the inclusions $\mathrm{T}^{2} \subset \mathrm{U}(2) \subset \mathrm{SU}(3)$ giving rise to the above homogeneous fibration. Here we embed $\mathrm{U}(2)$ as the upper $2 \times 2$ block, i.e., $\mathrm{U}(2)=\{\operatorname{diag}(A, \operatorname{det} \bar{A}) \mid A \in \mathrm{U}(2)\}$. A vector in $\mathfrak{m}$ is of the form $Y=\left(\begin{array}{cc}0 & v \\ -\bar{v} & 0\end{array}\right)$ with $v \in \mathbb{C}^{2}$ and one easily shows that $[A, Y]=A v+\operatorname{tr}(A) v$ for $A \in \mathfrak{u}(2)$. Hence if $X \in \mathfrak{p} \subset \mathfrak{s u}(2)$ and $Y \in \mathfrak{m},[X, Y]=0$ iff $X=0$ or $Y=0$. This shows that part (c) of Proposition 4.3 holds. As for $(a)$ and $(b)$ the fiber and base are symmetric spaces of rank 1 and thus $\mathrm{SU}(3) / \mathrm{T}^{2}$ has positive curvature. On the other hand, one easily sees that there are vectors $X, Y \in \mathfrak{m}+\mathfrak{p}$ with $[X, Y]=0$. Thus the biinvariant metric has non-negative curvature but with some 0 -curvature 2-planes. The Cheeger deformation deforms this metric into one with positive curvature.

2) The Berger space $B^{13}=\mathrm{SU}(5) / \mathrm{Sp}(2) \cdot \mathrm{S}^{1}$ admits a fibration

$$
\mathbb{R} \mathbb{P}^{5} \rightarrow \mathrm{SU}(5) / \mathrm{Sp}(2) \cdot \mathrm{S}^{1} \rightarrow \mathbb{C P}^{4},
$$

coming from the inclusions $\mathrm{Sp}(2) \cdot \mathrm{S}^{1} \subset \mathrm{U}(4) \subset \mathrm{SU}(5)$. Here $\mathrm{Sp}(2) \subset \mathrm{SU}(4)$ is the usual embedding and $\mathrm{S}^{1}$ is the center of $\mathrm{U}(4)$. Furthermore, the fiber is $\mathrm{U}(4) / \mathrm{Sp}(2) \cdot \mathrm{S}^{1}=\mathrm{SU}(4) / \mathrm{Sp}(2) \cdot \mathbb{Z}_{2}=\mathrm{SO}(6) / \mathrm{O}(5)=\mathbb{R} \mathbb{P}^{5}$. A biinvariant metric on $\mathrm{SU}(5)$ restricts to a biinvariant metric on $\mathrm{SO}(6)$ which induces a metric with constant curvature on the fiber $\mathbb{R} \mathbb{P}^{5}$. The base is clearly a symmetric space of rank 1 and condition (c) is verified as in the previous case.

3) The Aloff-Wallach spaces $W_{p, q}^{7}=\mathrm{SU}(3) / \operatorname{diag}\left(z^{p}, z^{q}, \bar{z}^{p+q}\right),(p, q)=$ 1 , form an infinite family. We claim that they have positive curvature iff $p q(p+q) \neq 0$. They admit a fibration

$$
\mathbb{S}^{3} / \mathbb{Z}_{p+q} \rightarrow W_{p, q} \rightarrow \mathrm{SU}(3) / \mathrm{T}^{2},
$$

coming from the inclusions $\operatorname{diag}\left(z^{p}, z^{q}, \bar{z}^{p+q}\right) \subset \mathrm{U}(2) \subset \mathrm{SU}(3)$. Hence, as long as $p+q \neq 0$, the fiber is the lens space $\mathrm{U}(2) / \operatorname{diag}\left(z^{p}, z^{q}\right)=\mathrm{SU}(2) / \operatorname{diag}\left(z^{p}, z^{q}\right)$ with $z^{p+q}=1$. 
A vector in $\mathfrak{m}$ again has the form $Y=\left(\begin{array}{cc}0 & v \\ -\bar{v} & 0\end{array}\right)$. Since the Lie algebra of $H$ is spanned by $\operatorname{diag}(i p, i q,-(i p+i q))$, we can write an element in $\mathfrak{p}$ as $X=\operatorname{diag}(A,-\operatorname{tr} A)$ where $A=\left(\begin{array}{cc}i(2 q+p) a & z \\ -\bar{z} & -i(q+2 p) a\end{array}\right)$ with $a \in \mathbb{R}$ and $z \in \mathbb{C}$. Hence $[X, Y]=A v+i(q-p) a v$, i.e., $A$ has an eigenvalue $i(p-q) a$ if $v \neq 0$. But one easily shows that this is only possible when $A=0$ or $p q=0$. Hence condition (c) is satisfied and since $(a)$ and $(b)$ clearly hold, $W_{p, q}$ has positive curvature as long as $p q(p+q) \neq 0$. If on the other hand one of $p, q$ or $p+q$ is 0 , say $p=0$, one easily shows that the fixed point set of $\operatorname{diag}(1,-1,-1) \in H=\operatorname{diag}\left(z^{p}, z^{q}, \bar{z}^{p+q}\right)=\operatorname{diag}(1, z, \bar{z})$ is equal to $\mathrm{U}(2) / \operatorname{diag}(z, \bar{z})=\mathbb{S}^{2} \times \mathbb{S}^{1} / \mathbb{Z}_{2}$. Since fixed point sets of isometries are totally geodesic, and since $\mathbb{S}^{2} \times \mathbb{S}^{1} / \mathbb{Z}_{2}$ does not carry a metric with positive curvature, these Aloff-Wallach spaces cannot carry a homogeneous metric with positive curvature.

4. Finally we have the Berger space: $B^{7}=\mathrm{SO}(5) / \mathrm{SO}(3)$. To describe the embedding $\mathrm{SO}(3) \subset \mathrm{SO}(5)$, we recall that $\mathrm{SO}(3)$ acts orthogonally via conjugation on the set of $3 \times 3$ symmetric traceless matrices. This space is special since $\mathrm{SO}(3)$ is maximal in $\mathrm{SO}(5)$ and hence does not admit a homogeneous fibration. It is also what is called isotropy irreducible, i.e., the isotropy action of $H$ on the tangent space is irreducible. This implies that there is only one $\mathrm{SO}(5)$ invariant metric up to scaling. Now a direct calculation is necessary in order to show that a biinvariant metric on $\mathrm{SO}(5)$ induces positive curvature on $B^{7}$.

Remark. a) The examples $B^{7}$ and $B^{13}$ were found by Berger [10] when classifying normal homogeneous metrics with positive curvature i.e., metrics on $G / H$ induced by a biinvariant metric on $G$. But in $[83]$ B. Wilking observed that the Aloff-Wallach space $W_{1,1}$ is missing since it can be written as $\mathrm{SU}(3) \mathrm{SO}(3) / \mathrm{U}(2)$ where a biinvariant metric induces positive curvature.

b) In [86] Wallach also proved that if one adds to the assumptions in Proposition 4.3 that the fiber is a symmetric pair as well, then the metrics $g_{t}$ with $1<t<4 / 3$ have positive curvature also. This applies to the flag manifolds and the Berger space $B^{13}$. We do not know of a simple geometric proof of this fact, similar to the one we gave in Proposition 4.3. It is also mysterious that the limiting value $4 / 3$ is the same as in Lemma 2.9. The number $4 / 3$ shows up again if one considers homogeneous metrics on spheres, scaled in the direction of one of the Hopf fibrations with fibers $\mathbb{S}^{1}, \mathbb{S}^{3}$ or $\mathbb{S}^{7}$. As was shown in $[\mathbf{8 1}]$, they have positive curvature as long as the scale is less than $4 / 3$. In the cases where the fiber is 3 or 7 dimensional, the proof again requires detailed curvature estimates. It would be interesting to obtain a uniform and less computational understanding why the number $4 / 3$ appears in all 3 cases. 
After a classification of all homogeneous spaces which admit a metric with positive curvature, one can ask for the best homogeneous metric, i.e., the one with largest pinching $\delta$. This is a rather difficult question since pinching constants are notoriously difficult to compute. For the homogeneous spaces which are not symmetric spaces of rank 1 this was done in [79] for the flag manifolds and in [67] for the remaining cases (see also $[26,47,50]$ for previous work). Interestingly, one obtains three homogeneous spaces, $B^{7}, B^{13}$ and $W_{1,1}$ which admit metrics with pinching $\delta=1 / 37$. In the first two cases this is the best metric and in the latter case the best one among all metrics invariant under $\mathrm{SU}(3) \mathrm{SO}(3)$. For the flag manifolds the best metric has pinching $\delta=1 / 64$. In [67] one finds numerical values for the pinching constants of the best homogeneous metrics on $W_{p, q} \neq W_{1,1}$. It turns out to be an increasing function of $p / q$ when $0<p \leq q$ and is in particular always $<1 / 37$. For $W_{1,1}$ the set of $\mathrm{SU}(3)$ invariant metrics is 10-dimensional, which makes the computations rather difficult, even numerically.

\section{Biquotients with positive curvature}

As explained in Section 2, biquotients $G / / H$ are obtained when $H \subset G \times G$ acts on $G$ from the left and from the right. When the action is free, the biinvariant metric on $G$ induces a metric on $G / / H$ with non-negative sectional curvature. In some cases, this can be deformed via a Cheeger deformation into one with positive curvature. We now describe these biquotient examples explicitly and prove that they have positive curvature.

1) There is an analogue of the 6-dimensional flag manifold which is a biquotient of $\mathrm{SU}(3)$ under an action of $\mathrm{T}^{2}=\{(z, w)|z, w \in \mathbb{C}| z,|=| w \mid=$ $1\}$. It is given by:

$$
E^{6}=\operatorname{diag}(z, w, z w) \backslash \mathrm{SU}(3) / \operatorname{diag}\left(1,1, z^{2} w^{2}\right)^{-1}
$$

The action by $\mathrm{T}^{2}$ is clearly free. In order to show that this manifold is not diffeomorphic to the homogeneous flag $W^{6}$, one needs to compute the cohomology with integer coefficients. The cohomology groups are the same for both manifolds, but the ring structure is different [28]. The fact that this manifold admits a metric with positive curvature will follow from the next example.

2) We now describe the 7-dimensional family of Eschenburg spaces $E_{k, l}$, which can be considered as a generalization of the Aloff Wallach spaces. Let $k:=\left(k_{1}, k_{2}, k_{3}\right)$ and $l:=\left(l_{1}, l_{2}, l_{3}\right) \in \mathbb{Z}^{3}$ be two triples of integers with $\sum k_{i}=\sum l_{i}$. We can then define a two-sided action of $\mathrm{S}^{1}=\{z \in \mathbb{C}|| z \mid=1\}$ on $\mathrm{SU}(3)$ whose quotient we denote by $E_{k, l}$ :

$$
E_{k, l}:=\operatorname{diag}\left(z^{k_{1}}, z^{k_{2}}, z^{k_{3}}\right) \backslash \mathrm{SU}(3) / \operatorname{diag}\left(z^{l_{1}}, z^{l_{2}}, z^{l_{3}}\right)^{-1}
$$


The action is free if and only if $\operatorname{diag}\left(z^{k_{1}}, z^{k_{2}}, z^{k_{3}}\right)$ is not conjugate to $\operatorname{diag}\left(z^{l_{1}}, z^{l_{2}}, z^{l_{3}}\right)$, i.e.

$$
\operatorname{gcd}\left(k_{1}-l_{i}, k_{2}-l_{j}\right)=1, \text { for all } i \neq j, i, j \in\{1,2,3\} \text {. }
$$

We now claim:

Proposition 4.4. An Eschenburg space $E_{k, l}$ has positive curvature if

$$
k_{i} \notin\left[\min \left(l_{1}, l_{2}, l_{3}\right), \max \left(l_{1}, l_{2}, l_{3}\right)\right]
$$

holds for all $1 \leq i \leq 3$.

Proof. As a metric we choose the one induced by a left invariant metric on $\mathrm{SU}(3)$, in fact the same one as in Lemma 4.2 that we used for $W^{6}$ and $W_{p, q}^{7}$. We first describe in a more explicit fashion the set of 0 -curvature 2 planes.

LEMMA 4.5. Let $Q_{t}$ be a left invariant metric on $\mathrm{SU}(3)$ as in Lemma 4.2 with $G=\mathrm{SU}(3)$ and $K=\mathrm{U}(2)=\operatorname{diag}(A, \operatorname{det} \bar{A})$. A 0-curvature 2-plane either contains a vector of the form $X=\operatorname{diag}(i, i,-2 i)$, which lies in the center of $\mathrm{U}(2)$, or one of the form $X=\operatorname{Ad}(k) \operatorname{diag}(-2 i, i, i)$ for some $k \in K$.

Proof. By Lemma 4.2 a 0-curvature 2-plane is spanned by $X, Y$ with $[X, Y]=\left[X_{\mathfrak{k}}, Y_{\mathfrak{k}}\right]=\left[X_{\mathfrak{p}}, Y_{\mathfrak{p}}\right]=0$. Since $X_{\mathfrak{p}}, Y_{\mathfrak{p}}$ are tangent to $G / K=\mathbb{C P}^{2}$, they are linearly dependent, and we can thus assume that $X_{\mathfrak{p}}=0$. If $X, Y$ both lie in $\mathfrak{k}$, the fact that $[X, Y]=0$ implies that the 2-plane intersects the center of $\mathfrak{u}(2) \cong \mathbb{R} \oplus \mathfrak{s u}(2)$, i.e. it contains $X=\operatorname{diag}(i, i,-2 i)$. If not, let $X=\operatorname{diag}(A,-\operatorname{tr} A)$ and $Y_{\mathfrak{p}}=\left(\begin{array}{cc}0 & v \\ -\bar{v} & 0\end{array}\right)$ with $0 \neq v \in \mathbb{C}^{2}$. Then $0=$ $\left[X, Y_{\mathfrak{p}}\right]=A v+(\operatorname{tr} A) v$ implies that $-\operatorname{tr} A$ and $2 \operatorname{tr} A$ are eigenvalues of $A$ which means $A$ is conjugate to $\operatorname{diag}(-2 i, i)$, which proves our claim.

In order to show that $Q_{t}$ induces positive curvature on $E_{k, l}$, we need to prove that a 0-curvature 2-plane can never be horizontal, i.e., it cannot be orthogonal to the vertical direction of the $\mathrm{S}^{1}$ action. Let $X_{1}=$ $i \operatorname{diag}\left(k_{1}, k_{2}, k_{3}\right)$ and $X_{2}=i \operatorname{diag}\left(l_{1}, l_{2}, l_{3}\right)$. Then the vertical space at $g \in$ $\mathrm{SU}(3)$ is spanned by $\left(R_{g}\right)_{*}\left(X_{1}\right)-\left(L_{g}\right)_{*}\left(X_{2}\right)$, where $R_{g}$ and $L_{g}$ are right and left translations. Since the metric is left invariant, we can translate horizontal and vertical space back to $e \in \mathrm{SU}(3)$ via $L_{g^{-1}}^{*}$. Thus the translated vertical space is spanned by $\operatorname{Ad}\left(g^{-1}\right) X_{1}-X_{2}$. We now need to show that a vector as in Lemma 4.5 can never be orthogonal to it.

To facilitate this computation, observe the following. If $\mathfrak{t}$ is the Lie algebra of a maximal torus in $G$, then critical points of the function $g \rightarrow$ $Q(\operatorname{Ad}(g) A, H)$ for fixed $A, H \in \mathfrak{t}$ are obtained when $\operatorname{Ad}(g) A \in \mathfrak{t}$ also. Indeed, if $g_{0}$ is critical, we have $0=Q\left(\left[Y, \operatorname{Ad}\left(g_{0}\right) A\right], H\right)=Q\left(\left[\operatorname{Ad}\left(g_{0}\right) A, H\right], Y\right)$ for all $Y \in \mathfrak{g}$ and thus $\left[\operatorname{Ad}\left(g_{0}\right) A, H\right]=0$. For a generic vector $H \in \mathfrak{t}$ we have that 
$\exp (t H)$ is dense in the compact torus $\exp (\mathfrak{t})$ and hence $\left[\operatorname{Ad}\left(g_{0}\right) A, \mathfrak{t}\right]=0$ which by maximality of $\mathfrak{t}$ implies that $\operatorname{Ad}\left(g_{0}\right) A \in \mathfrak{t}$. If $H$ is not generic, the claim follows by continuity.

We now apply this to the function $Q_{t}\left(\operatorname{Ad}(g) X_{1}-X_{2}, \operatorname{diag}(i, i,-2 i)\right)$ which we need to show is never 0 . This amounts to showing that $Q\left(\operatorname{Ad}(g) X_{1}\right.$, $\operatorname{diag}(i, i,-2 i)) \neq Q\left(\operatorname{diag}(i, i,-2 i), X_{2}\right)=l_{1}+l_{2}-2 l_{3}$. But maximum and minimum of the left hand side, according to the above observation, lies among the values $k_{r}+k_{s}-2 k_{t}, r, s, t$ distinct. Subtracting $\sum k_{i}=\sum l_{i}$ we see that one needs to assume that $l_{3} \notin\left[\min \left(k_{i}\right), \max \left(k_{i}\right)\right]$. Next, according to Lemma 4.5, we need $Q\left(\operatorname{Ad}(g) \operatorname{diag}(-2 i, i, i), X_{1}\right) \neq Q(\operatorname{Ad}(k) \operatorname{diag}(-2 i, i, i)$, $\left.X_{2}\right)$ ) for any $g \in G$ and $k \in K$. According to the above principle, the left hand side has max and min among $k_{r}+k_{s}-2 k_{t}$ whereas the right hand side among $-2 l_{1}+l_{2}+l_{3},-2 l_{2}+l_{1}+l_{3}$. Thus we need to assume that the interval $\left[\min \left(l_{1}, l_{2}\right), \max \left(l_{1}, l_{2}\right)\right]$ does not intersect $\left[\min \left(k_{i}\right), \max \left(k_{i}\right)\right]$. This is one of the possible cases. To obtain one of the other ones, we can choose a different block embedding for $K=\mathrm{U}(2) \subset \mathrm{SU}(3)$.

Among the biquotients $E_{k, l}$ there are two interesting subfamilies. $E_{p}=$ $E_{k, l}$ with $k=(1,1, p)$ and $l=(1,1, p+2)$ has positive curvature when $p>0$. It admits a large group acting by isometries. Indeed, $G=\mathrm{SU}(2) \times$ $\mathrm{SU}(2)$ acting on $\mathrm{SU}(3)$ on the left and on the right, acts by isometries in the Eschenburg metric and commutes with the $S^{1}$ action. Thus it acts by isometries on $E_{p}$ and one easily sees that $E_{p} / G$ is one dimensional, i.e., $E_{p}$ is cohomogeneity one. A second family consists of the cohomogeneity two Eschenburg spaces $E_{a, b, c}=E_{k, l}$ with $k=(a, b, c)$ and $l=(1,1, a+b+c)$. Here $c=-(a+b)$ is the subfamily of Aloff-Wallach spaces. The action is free iff $a, b, c$ are pairwise relatively prime and the Eschenburg metric has positive curvature iff, up to permutations, $a \geq b \geq c>0$ or $a \geq b>0, c<-a$. For these spaces $G=\mathrm{U}(2)$ acts by isometries on the right and $E_{a, b, c} / G$ is two dimensional. For a general Eschenburg space $G=\mathrm{T}^{3}$ acts by isometries and $E_{k, l} / G$ is four dimensional. In [39] it was shown that these groups $G$ are indeed the id component of the full isometry group of a positively curved Eschenburg space (unless it is an Aloff-Wallach space).

To see that the biquotient $\mathrm{SU}(3) / / \mathrm{T}^{2}$ has positive curvature, we can view it as an $\mathrm{S}^{1}$ quotient of the Eschenburg spaces $\operatorname{diag}\left(z^{p}, z^{q}, z^{p+q}\right) \backslash \mathrm{SU}(3) /$ $\operatorname{diag}\left(1,1, z^{2 p+2 q}\right)^{-1}$ which has positive curvature when $p q>0$.

3) We finally have the 13-dimensional Bazaikin spaces $B_{q}$, which can be considered as a generalization of the Berger space $B^{13}$. Let $q=\left(q_{1}, \ldots, q_{5}\right)$ be a 5 -tuple of integers with $q=\sum q_{i}$ and define

$$
B_{q}=\operatorname{diag}\left(z^{q_{1}}, \ldots, z^{q_{5}}\right) \backslash \mathrm{SU}(5) / \operatorname{diag}\left(z^{q}, A\right)^{-1},
$$

where $A \in \mathrm{Sp}(2) \subset \mathrm{SU}(4) \subset \mathrm{SU}(5)$. Here we follow the treatment in [89] of Bazaikin's work [3]. First, one easily shows that the action of $\operatorname{Sp}(2) \cdot S^{1}$ is 
free if and only if

$$
\text { all } q_{i} \text { 's are odd and } \operatorname{gcd}\left(q_{\sigma(1)}+q_{\sigma(2)}, q_{\sigma(3)}+q_{\sigma(4)}\right)=2 \text {, }
$$

for all permutations $\sigma \in S_{5}$. On SU(5) we choose an Eschenburg metric by scaling the biinvariant metric on $\mathrm{SU}(5)$ in the direction of $\mathrm{U}(4) \subset \mathrm{SU}(5)$. The right action of $\mathrm{Sp}(2) \cdot \mathrm{S}^{1}$ is then by isometries. Repeating the same arguments as in the previous case, one shows that the induced metric on $\mathrm{SU}(5) / / \mathrm{Sp}(2) \cdot \mathrm{S}^{1}$ satisfies

$$
\text { sec }>0 \text { if and only if } q_{i}+q_{j}>0(\text { or }<0) \text { for all } i<j \text {. }
$$

The special case of $q=(1,1,1,1,1)$ is the homogeneous Berger space. One again has a one parameter subfamily that is cohomogeneity one, given by $B_{p}=B_{(1,1,1,1,2 p-1)}$ since $\mathrm{U}(4)$ acting on the left induces an isometric action on the quotient. It has positive curvature when $p \geq 1$.

Unlike in the homogeneous case, there is no general classification of positively curved biquotients, except in the following cases. We call a metric on $G / / H$ torus invariant if it is induced by a left invariant metric on $G$ which is also right invariant under the action of a maximal torus. The main theorem in [28] states that an even dimensional biquotient $G / / H$ with $G$ simple and which admits a positively curved torus invariant metric is diffeomorphic to a rank one symmetric space or $\mathrm{SU}(3) / / T^{2}$. In the odd dimensional case he shows that $G / / H$ with a positively curved torus invariant metric and $G$ of rank 2 is either diffeomorphic to a homogeneous space or a positively curved Eschenburg space. In particular, the sufficient conditions in Proposition 4.4 are also necessary not only for Eschenburg metrics, but more generally torus invariant metrics. The classification of the remaining odd dimensional positively curved biquotients with $\operatorname{rk} G>2$ was taken up again in [12], where it was shown that if one assumes in addition that $H=H_{1} \cdot H_{2}$ with $H_{1}$ of rank one and such that $\mathrm{H}_{2}$ has no rank one factors and operates only on one side of $G$, the manifold is diffeomorphic to a homogeneous space, an Eschenburg space, or a Bazaikin space with positive curvature. The case where $G$ is not simple, on the other hand, is wide open. As we will see in Section 5, one obtains a large number of examples with almost positive curvature in this more general class of biquotients.

Not much is known about the pinching constants of the positively curved metrics on biquotients. One easily sees that for a sequences of Eschenburg spaces $E_{k, l}$ where $(k /|k|, l /|l|)$ converges to $((1,1,-2) / \sqrt{6},(0,0,0))$, the pinching of the Eschenburg metric converges to 1/37. In [25] W. Dickinson proved that for a general positively curved Eschenburg space $E_{k, l}$ with its Eschenburg metric one has $\delta \leq 1 / 37$ with equality only for $W_{1,1}$. Furthermore, the pinching constant for the cohomogeneity one Eschenburg spaces $E_{p}$ goes to 0 when $p \rightarrow \infty$. 


\section{Fundamental groups of positively curved manifolds}

A classical conjecture of S.S. Chern states that, analogously to the Preismann theorem for negative curvature, an abelian subgroup of the fundamental group of a positively curved manifold is cyclic. This is in fact not true. The first counter examples were given in $[\mathbf{7 1}]$, and further ones in $[\mathbf{3 8}]$ (see also $[4])$ :

THEOREM 4.6. The following groups act freely on a positively curved manifold:

(a) (Shankar) $\mathbb{Z}_{2} \oplus \mathbb{Z}_{2}$ on the Aloff Wallach space $W_{1,1}$ and the cohomogeneity one Eschenburg space $E_{2}$.

(b) (Grove-Shankar) The group $\mathbb{Z}_{3} \oplus \mathbb{Z}_{3}$ on the Aloff Wallach space $W_{p, q}$ if 3 does not divide $p q(p+q)$.

In the case of $W_{1,1}=G / H=\mathrm{SU}(3) / \operatorname{diag}\left(z, z, \bar{z}^{2}\right)$ this follows since $N(H) / H=\mathrm{U}(2) / Z(\mathrm{U}(2))=\mathrm{SO}(3)$ acts isometrically in the Eschenburg metric and the right action of $N(H) / H$ is free on any $G / H$. Thus a finite subgroup of $\mathrm{SO}(3)$ acts freely as well. Further example are given in [39] for the cohomogeneity two Eschenburg spaces. But there are no examples known where $\pi_{1}(M)=\mathbb{Z}_{p} \oplus \mathbb{Z}_{p}, p>3$ a prime, acts freely on a positively curved manifold.

\section{Topology of positively curved examples}

In dimension 7 and 13 we have infinitely many homotopy types of positively curved manifolds since an Eschenburg spaces satisfies $H^{4}\left(E_{k, l}, \mathbb{Z}\right)=$ $\mathbb{Z}_{r}$ with $r=\sigma_{2}(k)-\sigma_{2}(l)$ and for a Bazaikin spaces one has $H^{6}\left(B_{q}, \mathbb{Z}\right)=\mathbb{Z}_{r}$ with $8 r=\sigma_{3}(q)-\sigma_{1}(q) \sigma_{2}(q)$ where $\sigma_{i}$ is the elementary symmetric polynomial of degree $i$. On the other hand, for a fixed cohomology ring, there are only finitely many known examples $[\mathbf{2 0}, \mathbf{3 2}]$. A classification of 7 -dimensional manifolds whose cohomology type is like that of an Eschenburg space was obtained by Kreck-Stolz [58] in terms of certain generalized Eells-Kuiper invariants. They also computed these invariants for the Aloff Wallach spaces and obtained examples that are homeomorphic but not diffeomorphic. Kruggel [57] computed the Kreck-Stolz invariants for a general Eschenburg space in terms of number theoretic sums and Chinburg-Escher-Ziller [20] found further examples of this type.

THEOREM 4.7. One has the following examples with positive curvature:

(a) The pair of Aloff Wallach spaces $W_{k, l}$ with $(k, l)=(56.788,51.561)$ and $(k, l)=(61.213,18.561)$ and the pair of Eschenburg spaces $E_{k, l}$ with $(k ; l)=(79,49,-50 ; 0,46,32)$ and $(k ; l)=(75,54,-51$; $0,46,32)$ are homeomorphic to each other but not diffeomorphic.

(b) The pair of Aloff Wallach spaces $W_{k, l}$ with $(k, l)=$ (4.638.661, 4.056.005) and $(k, l)=(5.052 .965,2.458 .816)$ and the pair of 
Eschenburg spaces $E_{k, l}$ with $(k ; l)=(2.279,1.603,384 ; 0,0,4.266)$ and $(k ; l)=(2.528,939,799 ; 0,0,4.266)$ are diffeomorphic to each other but not isometric.

The diffeomorphic pairs of Aloff-Wallach spaces give rise to different components of the moduli space of positively curved metrics [59], in fact these two metrics cannot be connected even by a path of metrics with positive scalar curvature. The diffeomorphic pair of Eschenburg spaces are interesting since such cohomogeneity two manifolds also carry a 3-Sasakian metric (see Section 6) and they give rise to the first known manifold which carries two non-isometric 3-Sasakian metrics.

The situation for the Bazaikin spaces seems much more rigid. A computation of the Pontryagin classes and the linking form indicates, verified for the first 2 Billion examples, that Bazaikin spaces are all pairwise diffeomorphically distinct, see [32]. There is also only one Bazaikin space, the Berger space $B^{13}$, which is homotopy equivalent to a homogeneous space.

The Berger space $B^{7}=\mathrm{SO}(5) / \mathrm{SO}(3)$ plays a special role. It is, apart from spheres, the only known odd dimensional positively curved manifold which is 2-connected, which should be compared with the finiteness theorem by Fang-Rong and Petrunin-Tuschmann mentioned in Section 1. It is also, apart from the Hopf bundle, the only $\mathbb{S}^{3}$ bundle over $\mathbb{S}^{4}$ which is known to have positive curvature since it was shown in [35] that it is diffeomorphic to such a bundle. The topology of $\mathbb{S}^{3}$ bundles over $\mathbb{C P}^{2}$ is studied in $[31]$ where it is shown that they are frequently diffeomorphic to positively curved Eschenburg spaces when the bundle is not spin. It is thus natural to ask:

Problem 6. Does every $\mathbb{S}^{3}$ bundles over $\mathbb{S}^{4}$, and every $\mathbb{S}^{3}$ bundle over $\mathbb{C P}^{2}$ which is not spin, admit a metric with positive curvature. Do $\mathrm{S}^{3}$ principal bundles over $\mathbb{S}^{4}$, and $\mathrm{SO}(3)$-principal bundles over $\mathbb{C P}^{2}$ which are not spin, admit a metric with positive curvature invariant under the principal bundle action.

Notice that the existence in the latter case would imply infinitely many homotopy types of positively curved manifolds in dimension 6 . Also recall that there are two $\mathrm{SO}(3)$-principal bundles over $\mathbb{C P}^{2}$ with such positively curved metrics and that all bundles in Problem 6 have a metric with nonnegative curvature.

\section{Examples with almost positive or almost non-negative curvature}

As was suggested by Fred Wilhelm, there are two natural classes of metrics that lie in between non-negative curvature and positive curvature. In an initial step of deforming a non-negatively curved metric into one with positive curvature one can first make the curvature of all two planes at a point positive. We say that a metric has quasi positive curvature if there 
exists an open set such that all sectional curvatures in this open set are positive. In a second step one can try to deform the metric so that all sectional curvatures in an open and dense set are positive. We say that a metric with this property has almost positive curvature. It is natural to suggest that there should be obstructions to go from non-negative to quasi positive curvature and from almost positive to positive curvature, but that one should always be able to deform a metric from quasi positive to almost positive curvature.

The first example of a manifold with almost positive curvature was given by P.Petersen and F.Wilhelm in [66], where it was shown that $T_{1} \mathbb{S}^{4}$ has this property. In [82] it was shown that the Gromoll Meyer sphere $\Sigma^{7}=$ $\operatorname{Sp}(2) / / \operatorname{Sp}(1)$ admits a metric with almost positive curvature as well. See $[\mathbf{2 9}, \mathbf{3 0}]$ for a simpler proof for a slightly different metric on $\Sigma^{7}$.

We now describe some remarkable examples of metrics with almost positive curvature due to Wilking [84]:

THEOREM 5.1 (Wilking). Let $M$ be one of the following manifolds:

(a) One of the projectivised tangent bundles $P_{\mathbb{R}} T\left(\mathbb{R P}^{n}\right), P_{\mathbb{C}} T\left(\mathbb{C P}^{n}\right)$, or $P_{\mathbb{H}} T\left(\mathbb{H} \mathbb{P}^{n}\right)$.

(b) The homogeneous space $M_{p, q}^{4 n-1}=U(n+1) / H_{k, l}$ with $H_{k, l}=\{\operatorname{diag}$ $\left.\left(z^{p}, z^{q}, A\right)|| z \mid=1, A \in \mathrm{U}(n-1)\right\}$, where $p q<0$ and $(p, q)=1$.

Then $M$ carries a metric with almost positive curvature.

Here projectivised means that we identify a tangent vector $v$ with $\lambda v$ where $\lambda \in \mathbb{R}, \mathbb{C}$ or $\mathbb{H}$ respectively. Notice that in contrast to the known positively curved examples, these almost positively curved manifolds exist in arbitrarily high dimensions. Furthermore, in the case of $n=2$ these are one of the known manifolds with positive curvature, namely $P_{\mathbb{C}} T\left(\mathbb{C P}^{2}\right)$ and $P_{\mathbb{H}} T\left(\mathbb{H}^{2}\right)$ are the flag manifolds $W^{6}$ and $W^{12}$ and $M_{p, q}^{7}$ is the Aloff Wallach space $W_{p, q}$. Notice that the unique Aloff Wallach space $W_{1,0}=W_{1,-1}$ which does not admit a homogeneous metric with positive curvature, thus admits a metric with almost positive curvature.

These examples also show that in general a metric with almost positive curvature cannot be deformed to positive curvature everywhere. Indeed, $P_{\mathbb{R}} T\left(\mathbb{R} \mathbb{P}^{2 n+1}\right)$ is an odd dimensional non-orientable manifold and hence by Synge's theorem does not admit positive curvature. A particularly interesting special case is $P_{\mathbb{R}} T\left(\mathbb{R P}^{3}\right)=\mathbb{R} \mathbb{P}^{3} \times \mathbb{R P}^{2}$ and $P_{\mathbb{R}} T\left(\mathbb{R P}^{7}\right)=\mathbb{R P}^{7} \times \mathbb{R} \mathbb{P}^{6}$. If the manifold is compact and simply connected, it is not known whether an almost positively curved metric can be deformed to positive curvature. On the other hand, there either are obstructions, or the generalized Hopf conjecture on $\mathbb{S}^{3} \times \mathbb{S}^{2}$ is false.

Proof. We prove Theorem 5.1 in the simplest case of $P_{\mathbb{R}} T\left(\mathbb{R P}^{3}\right)=$ $\mathbb{R P}^{3} \times \mathbb{R P}^{2}$. 
Define a left invariant metric $g$ on $G=\mathrm{S}^{3} \times \mathrm{S}^{3}$ by scaling a biinvariant metric on $\mathfrak{g}$ in the direction of the diagonal subgroup $K=\Delta \mathrm{S}^{3}$ as in Lemma 4.2. Since $G / K=S^{3}$ is a symmetric pair of rank one, and since $(X, Y)_{\mathfrak{k}}=\frac{1}{2}(X+Y, X+Y)$ and $(X, Y)_{\mathfrak{m}}=\frac{1}{2}(X-Y,-X+Y)$, Lemma 4.2 implies that a 0 curvature plane is spanned by vectors $(u, 0),(0, u)$ with $0 \neq u \in \operatorname{Im} \mathbb{H}$. Here we regard $\mathrm{S}^{3}$ as the unit quaternions with Lie algebra $\operatorname{Im} \mathbb{H}$. $G$ acts on $T_{1} \mathbb{S}^{3}=\{(p, v)|| p|=| v \mid=1,\langle u, v\rangle=0\}$ via $\left(q_{1}, q_{2}\right) \star(p, v)=$ $\left(q_{1} p q_{2}^{-1}, q_{1} v q_{2}^{-1}\right)$ and the isotropy group of $(1, i)$ is equal to $H=\left(e^{i \theta}, e^{i \theta}\right)$ and thus $G / H=T_{1} \mathbb{S}^{3}$. We can rewrite the homogeneous space $G / H$ as a biquotient $\Delta G \backslash G \times G /(1 \times H)$ since $\Delta G \backslash G \times G=G$.

We now claim that the product metric $g+g$ on $G \times G$ induces a metric with almost positive curvature on $\Delta G \backslash G \times G /(1 \times H)$. For this, notice that each orbit of $\Delta G$ acting on the left on $G \times G$ contains points of the form $p=(\bar{a}, \bar{b}, 1,1), a, b \in \mathrm{S}^{3}$. The vertical space, translated to the identity via left translation with $(a, b, 1,1)$, is equal to the direct sum of $(A d(a) v, A d(a) w, v, w)$ with $v, w \in \operatorname{Im} \mathbb{H}$ and $(0,0, i, i) \cdot \mathbb{R}$. If we set $g(A, B)$ $=Q(P A, B)$ where $Q$ is a biinvariant metric on $G$, a horizontal vector is of the form $\left(P^{-1}(-A d(a) v,-A d(b) w), P^{-1}(v, w)\right)$ with $(v, w) \perp(i, i)$. Since $P$ clearly preserves 2 -planes spanned by $(v, 0),(0, v)$, a horizontal 0 -curvature plane is spanned by $\left(P^{-1}(-A d(a) v, 0), P^{-1}(v, 0)\right)$ and $\left(P^{-1}(0,-A d(b) v)\right.$, $\left.P^{-1}(0, v)\right)$ with $A d(a) v= \pm A d(b) v$. Thus $\bar{a} b$ either commutes or anticommutes with $v \in \operatorname{Im} \mathbb{H}$ and since also $v \perp i$, either $\bar{a} b \perp i$ or $\bar{a} b \perp 1$. Hence points with 0 -curvature 2 planes lie in two hypersurfaces in $G / H$.

Since the group $L$ generated by $(1,-1)$ and $(j, j)$ normalizes $H$, and since the left invariant metric $g$ is also right invariant under $L$, the quotient $G / H \cdot L$ inherits a metric with almost positive curvature and one easily sees that this quotient is $P_{\mathbb{R}} T\left(\mathbb{R} \mathbb{P}^{3}\right)=\mathbb{R} \mathbb{P}^{3} \times \mathbb{R} \mathbb{P}^{2}$.

See $[\mathbf{8 4}]$ for further examples. In [76] K. Tapp proved that the unit tangent bundles of $\mathbb{C P}^{n}, \mathbb{H} \mathbb{P}^{n}$ and $\mathrm{CaP}^{2}$, as well as the manifolds in Theorem 5.1 (b) with $p q>0$, have quasi positive curvature. In [56] M. Kerin showed that all Eschenburg spaces have a metric with quasi positive curvature and that $E_{0}$, the unique cohomogeneity one Eschenburg space which does not admit a cohomogeneity metric with positive curvature, admits a metric with almost positive curvature.

All known examples of almost positive curvature (and in fact all positively curved examples as well) can be described, after possibly enlarging the group, as so called normal biquotients, i.e., $M=G / / H$ with metric on $M$ induced by a biinvariant metric on $G$. B. Wilking showed in [84] that for such normal biquotients the exponential image of a 0-curvature 2-plane is totally geodesic and flat. As was observed by K. Tapp, this remains true more generally for a Riemannian submersion $G \rightarrow M$. It is a natural question to ask if the existence of an immersed flat 2-torus is sometimes an obstruction to deform a metric with non-negative curvature to one with positive curvature. 
In $[\mathbf{8 4}]$ one finds a number of natural open questions:

- Can every quasi positively curved metric be deformed to almost positive curvature.

- Can a quasi positively curved metric where the points with 0curvatures are contained in a contractible set be deformed to positive curvature.

- Does an even dimensional almost positively curved manifold have positive Euler characteristic.

\section{Almost non-negative curvature}

We say that a manifold $M$ has almost non-negative curvature if there exists a sequence of metrics $g_{i}$, normalized so that the diameter is at most 1 , with $\sec \left(g_{i}\right) \geq-1 / i$ for all $i \in \mathbb{N}$. This includes the almost flat manifolds where $\sec \left(g_{i}\right) \leq 1 / i$ as well. By Gromov's almost flat manifold theorem, the latter are finitely covered by a compact quotient of a nilpotent Lie group under a discrete subgroup.

This is a much larger class of manifolds. Besides being invariant under taking products, it is also well behaved under fibrations. In [34], FukayaYamaguchi showed that:

Theorem 5.2 (Fukaya-Yamaguchi). The total space of a principal Gbundle with $G$ compact over an almost non-negatively curved manifold is almost non-negatively curved as well.

To see this, one puts a metric on the total space $M$ such that the projection onto the base is a Riemannian submersion with totally geodesic fibers. Scaling the metric on $M$ in the direction of the fibers then has the desired properties as the scale goes towards 0 .

Thus every associated bundle $P \times_{G} F$, where $G$ acts isometrically on a non-negatively curved manifold $F$, has almost non-negative curvature as well. This applies in particular to all sphere bundles.

As was shown in [70], this class also includes all cohomogeneity one manifolds:

ThEOREM 5.3 (Schwachhöfer-Tuschmann). Every compact cohomogeneity one manifold has almost non-negative curvature.

Proof. As was observed by B. Wilking, this follows easily by using a Cheeger deformation. If a compact group $G$ acts by cohomogeneity one on $M$ then $M / G$ is either a circle or an interval. In the first case $M$ carries a $G$ invariant metric with non-negative curvature. In the second case we choose a $G$-invariant metric $g$ on $M$ which has non-negative curvature near the two singular orbits. This is clearly possible since a neighborhood of a singular orbit is a homogeneous disk bundle $G \times_{K} D$. We now claim that a Cheeger deformation $g_{t}$ with respect to the action of $G$ on $M$ has almost positive 
curvature as $t \rightarrow \infty$. Setting $s=1 / t$, the metric on $M=M \times G / \Delta G$ is induced by a metric of the form $g+s Q$ on $M \times G$. Thus its diameter is clearly bounded as $s \rightarrow 0$. On the regular part, we can assume that our 2-plane is spanned by vectors $X+\alpha T$ and $Y$, where $X, Y$ are tangent to a principal orbit and $T$ is a unit vector orthogonal to all principal orbits. As we saw in Section 2, the horizontal lift of these vectors to $M \times G$ under the Riemannian submersion $M \times G \rightarrow M$ are of the form $\left(s P^{-1}\left(s P^{-1}+\mathrm{Id}\right)^{-1} X+\right.$ $\left.\alpha T,-\left(s P^{-1}+\mathrm{Id}\right)^{-1} X\right)$ and $\left(s P^{-1}\left(s P^{-1}+\mathrm{Id}\right)^{-1} Y,-\left(s P^{-1}+\mathrm{Id}\right)^{-1} Y\right)$. Only the first component can contribute to a negative curvature. The curvature tensor of this component goes to 0 with $s$, of order 2 if $\alpha \neq 0$ and of order 4 if $\alpha=0$. On the other hand, $|(X+\alpha T) \wedge Y|_{g_{t}}^{2}$ goes to 0 with order 1 if $\alpha \neq 0$ and order 2 if $\alpha=0$. Hence the negative part goes to 0 as $s \rightarrow 0$.

The main obstruction theorems for almost non-negative curvature are:

- (Gromov) The Betti numbers are universally bounded in terms of the dimension.

- (Fukaya-Yamaguchi [34]) The fundamental group contains a nilpotent subgroup of finite index.

- (Kapovitch-Petrunin-Tuschmann [54]) A finite cover is a nilpotent space, i.e. the action of the fundamental group on its higher homotopy groups is nilpotent,

Notice that a compact quotient of a nilpotent non-abelian Lie group has almost non-negative curvature, but does not admit a metric with nonnegative curvature. On the other hand, for simply connected manifolds there are no known obstructions which could distinguish between almost nonnegative and non-negative (or even positive) curvature. As was suggested by $\mathrm{K}$. Grove, it is also natural to formulate the Bott conjecture more generally for almost non-negatively curved manifolds.

\section{Where to look for new examples?}

There are two natural suggestions where one might look for new examples with positive sectional curvature. The first is given by a structure that almost all known examples share. They are the total space of a Riemannian submersion. If one considers the more general class where the base space of the submersion is allowed to be an orbifold, then all known examples share this property, see [33].

\section{Fiber bundles}

A. Weinstein [87] considered fiber bundles $\pi: M \rightarrow B$, where $\pi$ is a Riemannian submersions with totally geodesic fibers. He called such a bundle fat if all vertizontal curvatures, i.e. the curvature of a 2-plane spanned by a horizontal and a vertical vector, are positive. This seemingly week 
assumption already places strong restrictions on the bundle. In fact, one has $[\mathbf{2 1}]$ :

THEOREM 6.1. (Derdzinski-Rigas) Every $\mathbb{S}^{3}$ bundle over $\mathbb{S}^{4}$ which is fat is a Hopf bundle.

This negative result seems to have discouraged the study of fat fiber bundles until recently. On the other hand, as we saw in Proposition 4.3, most homogeneous examples of positive curvature are the total space of a fat bundle. See [90] for a survey of what was known up to that point. In Theorem 6.7 we will see that there are infinitely many $\mathbb{S}^{3}$ orbifold bundles over $\mathbb{S}^{4}$ which are fat. It is thus natural and important to study fat bundles in this more general category.

A natural class of metrics is given by a connection metric on a principal $G$-bundle $\pi: P \rightarrow B$, sometimes also called a Kaluza Klein metric. Here one chooses a principal connection $\theta$, a metric $g$ on the base $B$, and a fixed biinvariant metric $Q$ on $G$ and defines:

$$
g_{t}(X, Y)=t Q(\theta(X), \theta(Y))+g\left(\pi_{*}(X), \pi_{*}(Y)\right) .
$$

The projection $\pi$ is then a Riemannian submersion with totally geodesic fibers isometric to $(G, t Q)$. Weinstein observed that the fatness condition is equivalent to requiring that the curvature $\Omega$ of $\theta$ has the property that $\Omega_{u}=Q(\Omega, u)$ is a symplectic 2 -form on the horizontal space for every $u \in \mathfrak{g}$. If $G=\mathrm{S}^{1}$, this is equivalent to the base being symplectic. If one wants to achieve positive curvature on the total space, we need to assume, in addition to the base having positive curvature, that $G=\mathrm{S}^{1}, \mathrm{SU}(2)$ or $\mathrm{SO}(3)$. In [17] a necessary and sufficient condition for positive curvature of such metrics was given. The proof carries over immediately to the category of orbifold principal bundles, which includes the case where the $G$ action on $P$ has only finite isotropy groups.

TheOREM 6.2. (Chaves-Derdzinski-Rigas) A connection metric $g_{t}$ on an orbifold $G$-principal bundle with $\operatorname{dim} G \leq 3$ has positive curvature, for $t$ sufficiently small, if and only if

$$
\left(\nabla_{x} \Omega_{u}\right)(x, y)^{2}<\left|i_{x} \Omega_{u}\right|^{2} k_{B}(x, y),
$$

for all linearly independent horizontal vectors $x, y$ and $0 \neq u \in \mathfrak{g}$.

Here $k_{B}(x, y)=g\left(R_{B}(x, y) y, x\right)$ is the unnormalized sectional curvature and $i_{x} \Omega_{u} \neq 0$ is precisely the above fatness condition. We call a principal connection with this property hyperfat.

The simplest examples of hyperfat principal connections are given by the Aloff-Wallach spaces $W_{k, l}$, considered as a circle bundle over $\mathrm{SU}(3) / T^{2}$, since the fibers of a homogeneous fibration are totally geodesic. As mentioned earlier, $W_{1,1}$ can also be considered as an $\mathrm{SO}(3)$ principal bundle over $\mathbb{C P}^{2}$ 
which thus carries an $\mathrm{SO}(3)$ hyperfat connection (a fact first observed in [16]). In the orbifold category one has many more examples. Recall that a metric is called 3-Sasakian if $\mathrm{SU}(2)$ acts isometrically and almost freely with totally geodesic orbits of curvature 1 . Moreover, for $U$ tangent to the $\mathrm{SU}(2)$ orbits and $X$ perpendicular to them, $X \wedge U$ is required to be an eigenvector of the curvature operator $\hat{R}$ with eigenvalue 1 . In particular the vertizontal curvatures are equal to 1 , i.e., the bundle is fat. This gives rise to a large new class of fat orbifold principal bundles, see $[\mathbf{1 3}]$ for a survey. The dimension of the base is a multiple of 4 , and its induced (orbifold) metric is quaternionic Kähler with positive scalar curvature. One easily sees that the condition on the curvature operator is equivalent to $\nabla_{x} \Omega_{u}=0$ for all $u \in \mathfrak{g}$. Hence we obtain the following Corollary, which was proved independently in $[\mathbf{2 2}]$ :

Corollary 6.3. A 3-Sasakian manifold has positive sectional curvature, after the metric in the direction of the $\mathrm{SU}(2)$ orbits is scaled down sufficiently, if and only if the quaternionic Kähler quotient has positive sectional curvature.

In [11] it was shown that a quaternionic Kähler manifold of dimension $4 n>4$ has positive sectional curvature if and only if it is isometric to $\mathbb{H}^{n}$, which also holds for orbifolds. When the base is 4-dimensional, quaternionic Kähler is equivalent to being self dual Einstein and here an interesting new family of examples arises. In [14] it was shown that the cohomogeneity two Eschenburg spaces $E_{a, b, c}=\operatorname{diag}\left(z^{a}, z^{b}, z^{c}\right) \backslash \mathrm{SU}(3) / \operatorname{diag}\left(1,1, z^{a+b+c}\right)$, with $a, b, c$ positive and pairwise relatively prime, carry a 3-Sasakian metric with respect to the right action by $\mathrm{SU}(2)$. The quotients are weighted projective spaces and Dearricott examined their sectional curvatures in [23]:

COROLlary 6.4. (Dearricott) The principal connection for the 3-Sasakian manifold $E_{a, b, c}$ with $0<a \leq b \leq c$ is hyperfat if and only if $c^{2}<a b$.

Although the total space also carries an Eschenburg metric with positive curvature, the projection to the base in that case does not have totally geodesic fibers.

Since many of the known examples are also the total space of sphere bundles, it is natural to study this category as well. A connection metric on a sphere bundle can be defined in terms of a metric connection $\nabla$ on the corresponding vector bundle. It induces a horizontal distribution on the sphere bundle and the fibers are endowed with a metric of constant curvature. An analogue of 6.2 for sphere bundles was proved in [75]:

THEOREM 6.5 (Tapp). A connection metric on an orbifold sphere bundle $E \rightarrow B$ has positive curvature, for sufficiently small radius of the fibers, if and only if

$$
\left\langle\left(\nabla_{x} R^{\nabla}\right)(x, y) u, v\right\rangle^{2}<\left|R^{\nabla}(u, v) x\right|^{2} k_{B}(x, y)
$$


for all linearly independent $x, y \in T_{p} B$ and $u, v \in E_{p}$, where we have set $\langle R(u, v) x, y\rangle=\langle R(x, y) u, v\rangle$

Notice that $R^{\nabla}(u, v) x \neq 0$ for all $u \wedge v \neq 0, x \neq 0$, means that $(x, y) \rightarrow\left\langle R^{\nabla}(x, y) u, v>\right.$ is nondegenerate for all $u \wedge v \neq 0$. But this is simply Weinstein's fatness condition for the sphere bundle.

Known examples are the homogeneous Wallach flag manifolds $W^{6}, W^{12}$, $W^{24}$. The Aloff-Wallach spaces $W_{p, q}$ with $p+q=1$ are hyperfat $\mathbb{S}^{3}$ bundles over $\mathbb{C P}^{2}$. Furthermore, the 3-Sasakian Eschenburg spaces $E_{a, b, c}$ in Corollary 6.4, where one of $a, b, c$ is even, are hyperfat $\mathbb{S}^{3}$ orbifold bundles and the associated $\mathbb{S}^{2}$ bundle $E_{a, b, c} / \mathrm{S}^{1} \rightarrow E_{a, b, c} / \mathrm{SU}(2)$ is hyperfat as well. If the base is a manifold, the condition $\nabla R=0$ is rather restrictive. For example, if the base is a symmetric space, it was shown in [45] that the bundle must be homogeneous. Homogeneous fat fiber bundles were classified in [8]. If the fiber dimension is larger than one, the base is symmetric. But if we assume in addition that the base has positive curvature, only the Wallach spaces remain.

The fiber bundle structure for most of the Eschenburg spaces and Bazaikin spaces do not have totally geodesic fibers. The Berger space $B^{7}=$ $\mathrm{SO}(5) / \mathrm{SO}(3)$ is also the total space of an $\mathrm{SU}(2)$ orbifold principal bundle over $\mathbb{S}^{4}$, but the fibers are again not totally geodesic. It is therefore also natural to examine warped connection metrics on the total space where the metric on the fiber is multiplied by a function on the base, see $[\mathbf{7 5}, \mathbf{7 2}]$. But notice that the known fibrations of the Eschenburg and Bazaikin spaces, with fiber dimension bigger than one, are not of this form either. Connection metrics with non-negative curvature have also been studied in $[\mathbf{7 3}, \mathbf{8 8}]$ and $[\mathbf{7 2}]$.

\section{Cohomogeneity one manifolds}

A second natural class of manifolds where one can search for new examples, especially in light of Theorem 4.1, are manifolds with low cohomogeneity. Positively curved homogeneous spaces are classified, so cohomogeneity one manifolds are the natural next case to study.

There are many cohomogeneity one actions on symmetric spaces of rank one. Among the examples of positive curvature discussed in Section 4, one has a number of other cohomogeneity one actions. As mentioned there, the positively curved Eschenburg spaces

$$
E_{p}^{7}=\operatorname{diag}\left(z, z, z^{p}\right) \backslash \mathrm{SU}(3) / \operatorname{diag}\left(1,1, \bar{z}^{p+2}\right), p \geq 1,
$$

and the Bazaikin spaces

$$
B_{p}^{13}=\operatorname{diag}\left(z, z, z, z, z^{2 p-1}\right) \backslash \mathrm{SU}(5) / \operatorname{Sp}(2) \operatorname{diag}\left(1,1,1,1, \bar{z}^{2 p+3}\right), p \geq 1,
$$

admit cohomogeneity one actions. Two further examples are the Wallach space $W_{1,1}^{7}=\mathrm{SU}(3) \mathrm{SO}(3) / \mathrm{U}(2)$ where $\mathrm{SO}(3) \mathrm{SO}(3)$ acts by cohomogeneity 
one, and the Berger space $B^{7}=\mathrm{SO}(5) / \mathrm{SO}(3)$ with $\mathrm{SO}(4) \subset \mathrm{SO}(5)$ acting by cohomogeneity one.

In even dimensions, L. Verdiani [80] classified all positively curved cohomogeneity one manifolds. Here only rank one symmetric spaces arise. In odd dimensions, one finds a "preclassification" in [41]. In dimension seven, a new family of candidates arises: Two infinite families $P_{k}, Q_{k}, k \geq 1$, and one isolated manifold $R$. The group diagram for $P_{k}$ is similar to the one considered in Theorem 2.10:

$$
H=\Delta Q \subset\left\{\left(e^{i \theta}, e^{i \theta}\right) \cdot H,\left(e^{j(1-2 k) \theta}, e^{j(1+2 k) \theta}\right) \cdot H\right\} \subset \mathrm{S}^{3} \times \mathrm{S}^{3},
$$

whereas the one for $Q_{k}$ is given by

$$
H=\{( \pm 1, \pm 1),( \pm i, \pm i)\} \subset\left\{\left(e^{i \theta}, e^{i \theta}\right) \cdot H,\left(e^{j k \theta}, e^{j(k+1) \theta}\right) \cdot H\right\} \subset \mathrm{S}^{3} \times \mathrm{S}^{3} .
$$

$R$ is similar to $Q_{k}$ with slopes $(3,1)$ on the left, and $(1,2)$ on the right.

THEOREM 6.6. (Verdiani, Grove-Wilking-Ziller) A simply connected cohomogeneity one manifold $M$ with an invariant metric of positive sectional curvature is equivariantly diffeomorphic to one of the following:

- An isometric action on a rank one symmetric space,

- One of $E_{p}^{7}, B_{p}^{13}$ or $B^{7}$,

- One of the 7-manifolds $P_{k}, Q_{k}$, or $R$,

with one of the actions described above.

The first in each sequence $P_{k}, Q_{k}$ admit an invariant metric with positive curvature since $P_{1}=\mathbb{S}^{7}$ and $Q_{1}=W_{1,1}^{7}$.

Among the cohomogeneity one manifolds with codimension 2 singular orbits, which all admit non-negative curvature by Theorem 2.5, are two families like the above $P_{k}$ and $Q_{k}$, but where the slopes for $K_{ \pm}$are arbitrary. It is striking that in positive curvature, with one exception, only the above slopes are allowed. The exception is given by the positively curved cohomogeneityone action on $B^{7}$, where the isotropy groups are like those for $P_{k}$ with slopes $(3,-1)$ and $(-1,3)$. In some tantalizing sense then, the exceptional Berger manifold $B^{7}$ is associated with the $P_{k}$ family in an analogues way as the exceptional candidate $R$ is associated with the $Q_{k}$ family. It is also surprising that all non-linear actions in the above Theorem, apart from the Bazaikin spaces $B_{p}^{13}$, are cohomogeneity one under a group locally isomorphic to $\mathrm{S}^{3} \times \mathrm{S}^{3}$.

These candidates also have interesting topological properties. $Q_{k}$ has the same cohomology groups as $E_{k}$ with $H^{4}\left(Q_{k}, \mathbb{Z}\right)=\mathbb{Z}_{k}$. The manifolds $P_{k}$ are all 2-connected with $H^{4}\left(P_{k}, \mathbb{Z}\right)=\mathbb{Z}_{2 k-1}$. Thus it is natural to ask:

Problem 7. Are any of the manifolds $Q_{k}, k>1$, diffeomorphic to a positively curved Eschenburg space? Are any of the manifolds $P_{k}, k>1$, diffeomorphic to an $\mathbb{S}^{3}$ bundle over $\mathbb{S}^{4}$ ? 
Manifolds of this type are classified by their Kreck-Stolz invariants, but these can be very difficult to compute in concrete cases.

A somewhat surprising property that these candidates also have is that they admit fat principal connections, in fact they admit 3-Sasakian metrics:

THEOREM 6.7. $P_{k}$ and $Q_{k}$ admit 3-Sasakian metrics which are orbifold $\mathrm{S}^{3}$-principal bundles over $\mathbb{S}^{4}$ respectively $\mathbb{C P}^{2}$.

This follows [41] from the celebrated theorem due to Hitchin [48] that $\mathbb{S}^{4}$ admits a family of self dual Einstein orbifold metrics invariant under the cohomogeneity one action by $\mathrm{SO}(3)$, one for each $k \geq 1$, which is smooth everywhere except normal to one of the singular orbits where it has angle $2 \pi / k$. One then shows that the induced 7 -dimensional 3-Sasakian metric has no orbifold singularities, and by comparing the isotropy groups of the cohomogeneity one actions, it follows that they are equivariantly diffeomorphic to $P_{k}$ and $Q_{k}$. Unfortunately, the self dual Einstein metric on the base does not have positive curvature, unless $k=1$, corresponding to the smooth metrics on $\mathbb{S}^{4}$ respectively $\mathbb{C P}^{2}$. So Corollary 6.3 does not easily yield the desired metrics of positive curvature on $P_{k}$ and $Q_{k}$.

Hence these candidates surprisingly have both features, they admit cohomogeneity one actions, and are also the total space of an orbifold principal bundle. Both of these properties thus suggest concrete ways of finding new metrics with positive curvature. We thus end with our final problem.

Problem 8. Do all manifolds $P_{k}, Q_{k}$ and $R$ admit a cohomogeneity metric with positive curvature?

Notice that a positive answer for the manifolds $P_{k}$ would give infinitely many homotopy types of positively curved 2-connected manifolds. Thus the pinching constants $\delta_{k}$, for any metric on $P_{k}$, would necessarily go to 0 as $k \rightarrow \infty$, and $P_{k}$ would be the first examples of this type. It is natural to suggest that the manifolds $E_{p}$, although not 2-connected, should have the same property since the pinching constant for the Eschenburg metric goes to 0 as $p \rightarrow \infty$.

\section{References}

[1] A.V. Alekseevsy and D.V. Alekseevsy, G-manifolds with one dimensional orbit space, Ad. in Sov. Math. 8 (1992), 1-31.

[2] S. Aloff and N. Wallach, An infinite family of 7-manifolds admitting positively curved Riemannian structures, Bull. Amer. Math. Soc. 81(1975), 93-97.

[3] Y. Bazaikin, On a family of 13-dimensional closed Riemannian manifolds of positive curvature, Siberian Math. J. 37 (1996), 1068-1085.

[4] Y. Bazaikin, A manifold with positive sectional curvature and fundamental group $Z_{3} \oplus Z_{3}$, Siberian Math. J. 40 (1999), 834-836.

[5] I. Belegradek, Vector bundles with infinitely many souls, Proc. Amer. Math. Soc. 131 (2003), 2217-2221. 
[6] I. Belegradek and V. Kapovitch, Topological obstructions to nonnegative curvature, Math. Ann. 320 (2001), 167-190.

[7] I. Belegradek and V. Kapovitch, Obstructions to nonnegative curvature and rational homotopy theory, J. Amer. Math. Soc. 16 (2003), 259-284 .

[8] L. Bérard-Bergery, Sur certaines fibrations d'espaces homogènes riemanniens, Compositio Math 30 (1975), 43-61.

[9] L. Bérard Bergery, Les variétés riemanniennes homogènes simplement connexes de dimension impaire à courbure strictement positive, J. Math. pure et appl. 55 (1976), 47-68.

[10] M. Berger, Les variétés riemanniennes homogènes normales simplement connexes à courbure strictement positive, Ann. Scuola Norm. Sup. Pisa 15 (1961), 179-246.

[11] M. Berger, Trois remarques sur les variété riemanniennes à courbure positive, C.R. Acad. Sci. Paris 263 (1966), 76-78.

[12] R. Böck, Doppelquotienten ungerader dimension und positive Schnittkrümung, Dissertation, University of Augsburg, 1998.

[13] C.P. Boyer and K. Galicki 3-Sasakian manifolds, Surveys in differential geometry: essays on Einstein manifolds, Surv. Differ. Geom. VI (1999), 123-184.

[14] C.P. Boyer, K. Galicki and B. M. Mann The Geometry and Topology of 3-Sasakian Manifolds, J. für die reine und angew. Math. 455 (1994), 183-220.

[15] N. Brown, R. Finck, M. Spencer, K. Tapp and Z. Wu, Invariant metrics with nonnegative curvature on compact Lie groups, Can. Math. Bull., to appear.

[16] L.M. Chaves, A theorem of finiteness for fat bundles, Topology 33 (1994), 493-497.

[17] L. Chaves, A. Derdzinski and A. Rigas A condition for positivity of curvature, Bol. Soc. Brasil. Mat. 23 (1992), 153-165.

[18] J. Cheeger, Some examples of manifolds of nonnegative curvature, J. Diff. Geom. 8 (1973), 623-628.

[19] J. Cheeger and D. Gromoll, On the structure of complete manifolds of nonnegative curvature, Ann. of Math. 96 (1972), 413-443.

[20] T. Chinburg, C. Escher, and W. Ziller, Topological properties of Eschenburg spaces and 3-Sasakian manifolds, Math. Ann. 339 (2007), 3-20.

[21] A. Derdzinski and A. Rigas. Unflat connections in 3-sphere bundles over $\mathbb{S}^{4}$, Trans. of the AMS, 265 (1981), 485-493.

[22] O. Dearricott, Positive sectional curvature on 3-Sasakian manifolds, Ann. Global Anal. Geom. 25 (2004), 59-72.

[23] O. Dearricott, Positively curved self-dual Einstein metrics on weighted projective spaces, Ann. Global Ana. Geom. 27 (2005), 79-86.

[24] A. Dessai and W. Tuschmann, Nonnegative curvature and cobordism type, Math. Zeitschrift 257 (2007), 7-12.

[25] W. Dickinson, Curvature properties of the positively curved Eschenburg spaces, Diff. Geom. and its Appl. 20 (2004), 101-124.

[26] H.J. Eliasson, Die Krümmung des Raumes Sp(2)/SU(2) von Berger, Math. Ann. 164 (1966), 317-323.

[27] J.H. Eschenburg, New examples of manifolds with strictly positive curvature, Invent. Math. 66 (1982), 469-480.

[28] J.H. Eschenburg, Freie isometrische Aktionen auf kompakten Lie-Gruppen mit positiv gekrümmten Orbiträumen, Schriftenr. Math. Inst. Univ. Münster 32 (1984).

[29] J.H. Eschenburg, Almost positive curvature on the Gromoll-Meyer 7-sphere, Proc. Amer. Math. Soc. 130 (2002), 1165-1167.

[30] J.H. Eschenburg and M. Kerin, Almost positive curvature on the Gromoll-Meyer 7-sphere (corrected), Preprint 2007.

[31] C. Escher and W. Ziller, On the topology of non-negatively curved manifolds, in preparation. 
[32] L.A. Florit and W. Ziller, On the topology of positively curved Bazaikin spaces, J. Europ. Math. Soc., to appear.

[33] L.A. Florit and W. Ziller, Orbifold fibrations of Eschenburg spaces, Geom. Ded., to appear.

[34] K. Fukaya and T. Yamaguchi, The fundamental group of almost nonnegatively curved manifolds, Ann. of Math. 136 (1992), 253-333.

[35] S. Goette, N. Kitchloo and K. Shankar, Diffeomorphism type of the Berger space SO(5)/SO(3), Amer. Math. J. 126 (2004), 395-416.

[36] D. Gromoll and W. Meyer, An exotic sphere with nonnegative sectional curvature, Ann. of Math. (2) 100 (1974), 401-406.

[37] K. Grove and S. Halperin, Contributions of rational homotopy theory to global problems in geometry, Publ. Math. I.H.E.S. 56 (1982), 171-177.

[38] K. Grove and K. Shankar, Rank two fundamental groups of positively curved manifolds, J. Geometric Analysis, 10 (2000), 679-682.

[39] K. Grove, R. Shankar and W. Ziller, Symmetries of Eschenburg spaces and the Chern problem, Special Issue in honor of S. S. Chern, Asian J. Math. 10 (2006), 647-662.

[40] K. Grove, L. Verdiani, B. Wilking and W. Ziller, Non-negative curvature obstruction in cohomogeneity one and the Kervaire spheres, Ann. del. Scuola Norm. Sup. 5 (2006), 159-170.

[41] K. Grove, B. Wilking and W. Ziller, Positively curved cohomogeneity one manifolds and 3-Sasakian geometry, J. Diff. Geom., to appear.

[42] K. Grove and W. Ziller, Curvature and symmetry of Milnor spheres, Ann. of Math. 152 (2000), 331-367.

[43] K. Grove and W. Ziller, Cohomogeneity one manifolds with positive Ricci curvature, Inv. Math. 149 (2002), 619-646.

[44] K. Grove and W. Ziller, Lifting group actions and nonnegative curvature, Preprint 2007.

[45] L. Guijarro, L. Sadun, and G. Walschap. Parallel connections over symmetric spaces, J. Geom. Anal. 11 (2001), 265-281.

[46] R.S. Hamilton, Three-manifolds with positive Ricci curvature, J. Diff. Geom. 17 (1982), 255-306.

[47] E. Heintze, The curvature of $\mathrm{SU}(5) / \mathrm{Sp}(2) \mathrm{S} 1$, Invent. Math. 13 (1971), 205-212.

[48] N. Hitchin, A new family of Einstein metrics, Manifolds and geometry (Pisa, 1993), 190-222, Sympos. Math., XXXVI, Cambridge Univ. Press, Cambridge, 1996.

[49] C. Hoelscher, Cohomogeneity one manifolds in low dimensions, Ph.D. thesis, University of Pennsylvania, 2007.

[50] H.-M. Huang, Some remarks on the pinching problems, Bull. Inst. Math. Acad. Sin. 9 (1981), 321-340.

[51] J. Huizenga and K. Tapp, Invariant metrics with nonnegative curvature on $\mathrm{SO}(4)$ and other Lie groups, Mich. Math. J., to appear.

[52] W.Y. Hsiang and B. Lawson, Minimal submanifolds of low cohomogeneity, J. Diff. Geom. 5 (1971), 1-38.

[53] V. Kapovitch, A. Petrunin and W. Tuschmann, Nonnegative pinching, moduli spaces and bundles with infinitely many souls, J. of Diff. Geometry 71 (2005), 365-383.

[54] V. Kapovitch, A. Petrunin and W. Tuschmann, Nilpotency, almost nonnegative curvature and the gradient push, Ann. of Math., to appear.

[55] V. Kapovitch-W. Ziller, Biquotients with singly generated rational cohomology, Geom. Dedicata 104 (2004), 149-160.

[56] M. Kerin, Biquotients with almost positive curvature, Ph.D. thesis, University of Pennsylvania, in preparation.

[57] B. Kruggel, Homeomorphism and diffeomorphism classification of Eschenburg spaces, Quart. J. Math. Oxford Ser. (2) 56 (2005), 553-577. 
[58] M. Kreck and S. Stolz, Some non diffeomorphic homeomorphic homogeneous 7-manifolds with positive sectional curvature, J. Diff. Geom. 33 (1991), 465-486.

[59] M. Kreck and S. Stolz, Non connected moduli spaces of positive sectional curvature metrics, J. Amer. Math. Soc. 6 (1993), 825-850.

[60] S. López de Medrano, Involutions on Manifolds, Springer-Verlag, Berlin, 1971.

[61] P. Müter, Krümmungserhöhende Deformationen mittels Gruppenaktionen, Ph.D. thesis, Univerity of Münster, 1987.

[62] M. Özaydin and G. Walschap, Vector bundles with no soul, Proc. Amer. Math. Soc. 120 (1994), 565-567.

[63] R.S. Palais and T.E. Stewart, The cohomology of differentiable transformation groups, Am. J. Math. 83 (1961), 623-644.

[64] J. Parker, 4-dimensional G-manifolds with 3-dimensional orbits, Pacific J. Math. 125 (1986), 187-204.

[65] G.P. Paternain and J. Petean, Minimal entropy and collapsing with curvature bounded from below, Inv. Math. 151 (2003), 415-450.

[66] P. Petersen and F. Wilhelm, Examples of Riemannian manifolds with positive curvature almost everywhere, Geom. Topol. 3 (1999), 331-367.

[67] T. Püttmann, Optimal pinching constants of odd dimensional homogeneous spaces, Invent. math. 138, (1999), 631-684.

[68] A. Rigas, Geodesic spheres as generators of the homotopy groups of O, BO, J. Differential Geom. 13 (1978), 527-545.

[69] L. Schwachhöfer, Lower curvature bounds and cohomogeneity one manifolds, Differential Geom. Appl. 17 (2002), 209-228.

[70] L. Schwachhöfer and W. Tuschmann, Metrics of positive Ricci curvature on quotient spaces, Math. Ann. 330 (2004), 59-91.

[71] K. Shankar, On the fundamental groups of positively curved manifolds, J. Diff. Geom. 49 (1998), 179-182.

[72] K. Shankar, K. Tapp, and W. Tuschmann, Nonnegatively and positively curved metrics on circle bundles, Proc. of the AMS, 133 (2005), 2449-2459.

[73] M. Strake and G. Walschap, Connection metrics of nonnegative curvature on vector bundles, Manuscr. Math., 66 (1990), 309-318.

[74] E. Straume, Compact connected Lie transformation groups on spheres with low cohomogeneity. I,II, Mem. Amer. Math. Soc. 119 (1996), no. 569; 125 (1997), no. 595.

[75] K. Tapp, Conditions for nonnegative curvature on vector bundles and sphere bundles, Duke Math. J. 116 (2003), 77-101.

[76] K. Tapp, Quasipositive curvature on homogeneous bundles, J. Diff. Geom. 65 (2003), $273-287$.

[77] B. Totaro, Cheeger manifolds and the classification of biquotients, J. Diff. Geom. 61 (2002), 397-451.

[78] B. Totaro, Curvature, diameter, and quotient manifolds, Math. Res. Lett. 10 (2003), 191-203.

[79] F.M. Valiev, Precise estimates for the sectional curvatures of homogeneous Riemannian metrics on Wallach spaces, Sib. Mat. Zhurn. 20 (1979), 248-262.

[80] L. Verdiani, Cohomogeneity one manifolds of even dimension with strictly positive sectional curvature, J. Diff. Geom. 68 (2004), 31-72.

[81] L. Verdiani, W. Ziller, Positively curved homogeneous metrics on spheres, Preprint 2007.

[82] F. Wilhelm, An exotic sphere with positive curvature almost everywhere, J. Geom. Anal. 11 (2001), 519-560.

[83] B. Wilking, The normal homogeneous space $\mathrm{SU}(3) \mathrm{SO}(3) / \mathrm{U}(2)$ has positive sectional curvature, Proc. Amer. Soc. 127 (1999), 1191-1194.

[84] B. Wilking, Manifolds with positive sectional curvature almost everywhere, Inv. Math. 148 (2002), 117-141. 
[85] B. Wilking, Positively curved manifolds with symmetry, Ann. of Math. 163 (2006), 607-668. appear.

[86] N. Wallach, Compact homogeneous Riemannian manifolds with strictly positive curvature, Ann. of Math. 96 (1972), 277-295.

[87] A. Weinstein, Fat bundles and symplectic manifolds, Adv. in Math. 37 (1980), 239-250.

[88] D. Yang, On complete metrics of nonnegative curvature on 2-plane bundles, Pacific J. Math. 171 (1995), 569-583.

[89] W. Ziller, Homogeneous spaces, biquotients, and manifolds with positive curvature, Lecture Notes 1998, unpublished.

[90] W. Ziller, Fatness revisited, Lecture Notes 2000, unpublished.

University of Pennsylvania, Philadelphia, PA 19104

E-mail address: wziller@math.upenn.edu 